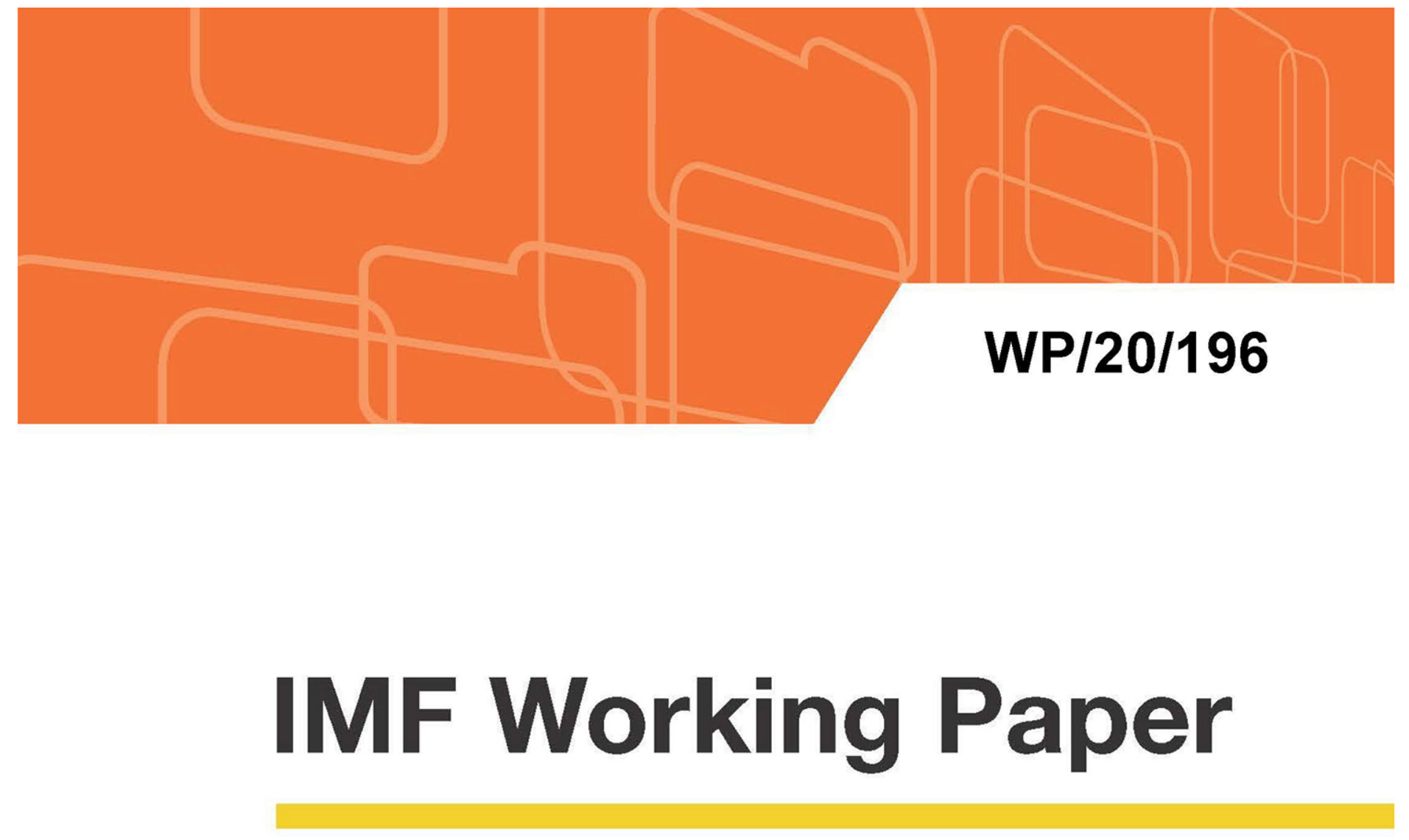

\title{
Should Inequality Factor into Central Banks' Decisions?
}

by Niels-Jakob H. Hansen, Alessandro Lin, and Rui C. Mano

IMF Working Papers describe research in progress by the author(s) and are published to elicit comments and to encourage debate. The views expressed in IMF Working Papers are those of the author(s) and do not necessarily represent the views of the IMF, its Executive Board, or IMF management. 


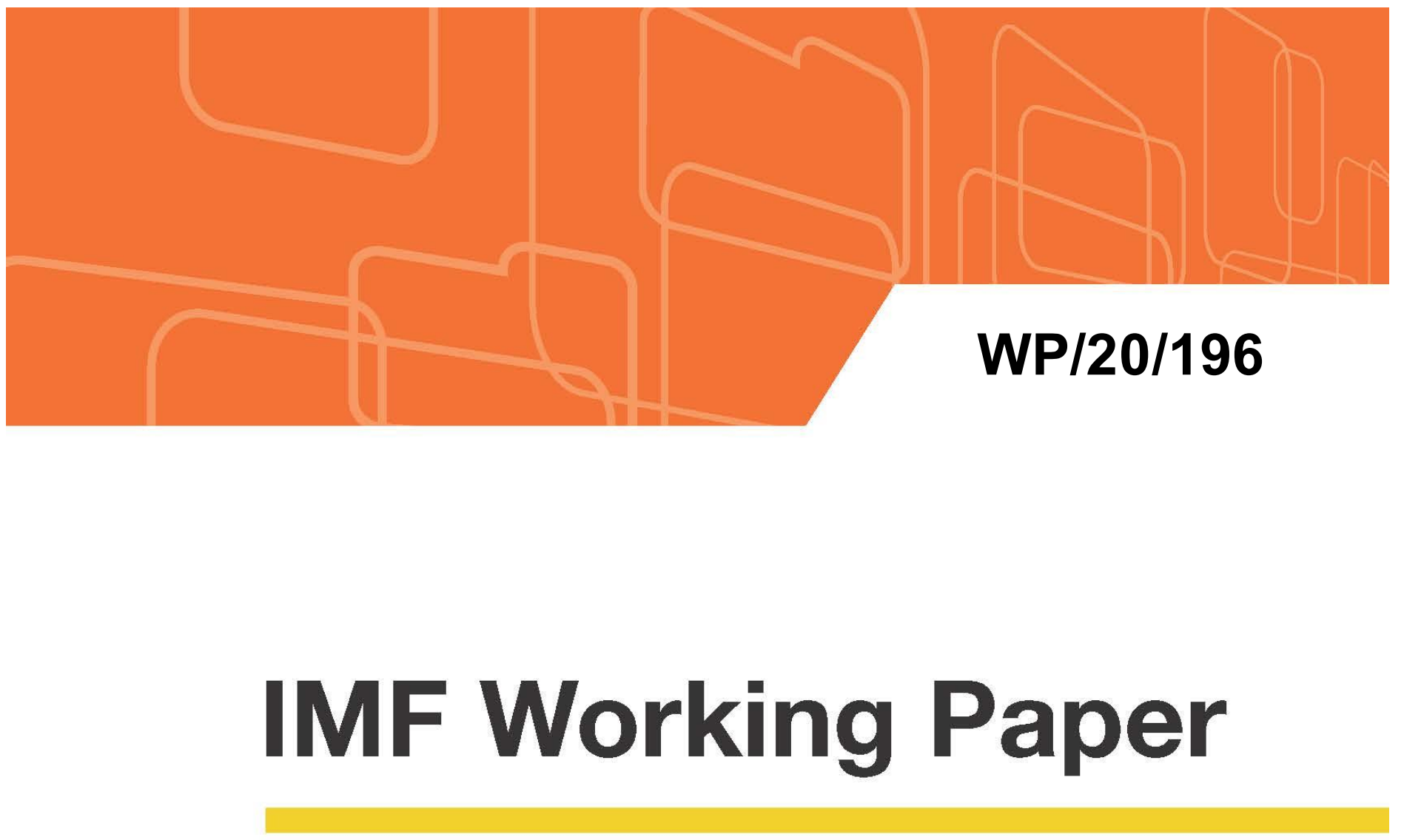

\section{Should Inequality Factor into Central Banks' Decisions?}

by Niels-Jakob H. Hansen, Alessandro Lin, and Rui C. Mano

IMF Working Papers describe research in progress by the author(s) and are published to elicit comments and to encourage debate. The views expressed in IMF Working Papers are those of the author(s) and do not necessarily represent the views of the IMF, its Executive Board, or IMF management. 


\title{
IMF Working Paper
}

Strategy, Policy and Review Department

\author{
Should Inequality Factor into Central Banks' Decisions? \\ Prepared by Niels-Jakob H. Hansen, Alessandro Lin, and Rui C. Mano ${ }^{1}$ \\ Authorized for distribution by Rupa Duttagupta
}

September 2020

\section{IMF Working Papers describe research in progress by the author(s) and are published to elicit comments and to encourage debate. The views expressed in IMF Working Papers are those of the author(s) and do not necessarily represent the views of the IMF, its Executive Board, or IMF management.}

\begin{abstract}
Inequality is increasingly a concern. Fiscal and structural policies are well-understood mitigators. However, less is known about the potential role of monetary policy. This paper investigates how inequality matters for monetary policy within a tractable Two-Agent New Keynesian model that captures important dimensions of inequality. We find some support for making inequality an explicit target for monetary policy, particularly if central banks follow standard Taylor rules.
\end{abstract}

JEL Classification Numbers: E21, E32, E52.

Keywords: Inequality, Optimal monetary policy, Taylor rules.

Author’s E-Mail Address: nhansen@imf.org; alessandro_lin@brown.edu; rmano@imf.org

\footnotetext{
${ }^{1}$ We havebenefited from insightful comments by Pierpaolo Benigno, Nina Biljanovska, Gauti Eggertsson, Jesper Lindé, Gurnain Pasricha, Adrian Peralta-Alva, Ricardo Reis, Xin Tang, Marina Tavares and Carl Walsh and participants in various internal IMF seminars, and the Brown Macro Lunch Seminar. We a lso thank Valentina Bonifacio for sharing SCF data. All remaining errors are our own.
} 


\section{Introduction}

Should central banks care about inequality when setting monetary policy? Up until recently this question was a non-starter among policy makers and academics. First, inequality is typically outside central banks' mandates. Second, an early literature showed that inequality of wealth or income did not distort the aggregate transmission of shocks. However, major central bank officials are increasingly discussing distributional issues. ${ }^{1}$ At the same time, advances in economic theory have shed light on the role of inequality in the transmission of monetary policy.

In this paper, we investigate how inequality affects desirable monetary policy in a stylized model where the economy is subject to technology shocks. The model is an extension of Debortoli and Gali, 2017. In this setting, a rich agent owns all the capital and thus her income is composed of after-tax dividends and wages. In contrast, a poor agent receives only wages and potentially a transfer from the government financed by the dividend tax. Positive productivity shocks lead to higher profits and hence higher capital income, thereby exacerbating initial income and consumption inequities. Moreover, we assume wage income is subject to tech-bias: when productivity rises, the rich agent's share of total wage income goes up, while the poor agent's declines. These mechanisms are consistent with important features of U.S. microand macro-economic data and match the empirical effects of technology shocks on consumption inequality (De Giorgi and Gambetti, 2017). The great advantage of this simple setting is that it allows studying how optimal monetary policy varies with inequality in a model with closed form solutions. That advantage comes at a cost of assuming a simplistic wealth distribution in steady-state and no aggregate savings in equilibrium. We also abstract from heterogeneity in the extensive margin of labor which is known to be another important driver of inequality over the cycle, although the assumption of tech-biased wage income could be thought of as a reduced form of that channel. ${ }^{2}$

We study implications for monetary policy under both optimal policy (Rotemberg and Woodford, 1997) and augmented Taylor rules (Taylor, 1993).

- Under optimal policy, the central bank chooses the welfare maximizing path of interest rates with full information and caring equally about all individuals. We find that such a central bank chooses to place some weight on observed consumption inequality, as that improves welfare. However, the optimal weight on consumption inequality is small compared to those on output and inflation gaps. Moreover, a central bank pursuing such optimal policy cares progressively less about inflation and more about growth the higher the initial level of inequality. This is because in an economy with high inequality, stabilizing inequality coincides with stabilizing growth, since the poor depend more on wages the greater the inequality, and wages are more stable when growth is stable.

- Under standard Taylor rules, the central bank chooses the interest rate based on the state of the aggregate economy, namely current output and the inflation gap. We find that central banks should also target consumption inequality through an "augmented" Taylor rule. Under such a rule, inter-

\footnotetext{
${ }^{1}$ As examples, see a recent speech by Chair Jerome H. Powell, that links the adjustment to the employment mandate of the Federal Reserve with an appreciation for benefits to low- and moderate-income communities, or an older speech by Chair Janet L. Yellen in 2014 and a speech by ECB President Mario Draghi in 2016.

${ }^{2} \mathrm{~A}$ separate but related literature on the effects of monetary policy shocks on wealth and income inequality has developed recently, see e.g Dolado et al., forthcoming and Coibion et al., 2017. In this paper, we focus on how the initial level of consumption inequality matters for the best monetary policy responses to a technology shock. We focus on consumption inequality as this concept features in the social welfare framework that we use to evaluate monetary policy.
} 
est rates should be set lower than otherwise following a positive technology shock. A policy of lower interest rates leads to higher wages, which benefits poor agents. Beyond lowering inequality, such a policy is also beneficial more generally because it improves inflation and growth outcomes by avoiding excessive monetary tightening in response to a positive productivity shock. These insights carry through in the presence of price and wage rigidities.

Literature review. Our paper relates to three literature strands: (i) Two-Agent models in a New Keynesian setup; (ii) optimal monetary policy; and (iii) the interplay of inequality and monetary policy.

First, we use a Two-Agent New Keynesian model (TANK). An early example of a TANK is Campbell and Mankiw, 1989 where one agent is forward looking and consumes according to her permanent income, while the other agent follows a "rule of thumb" of consuming only her current income. Gali et al., 2007 introduce these two types of consumers in a New Keynesian framework with price rigidities. Other examples of two-agent models in the New Keynesian environment include Bilbiie, 2008 and Bilbiie and Straub, 2013, who introduce heterogeneity by varying the degree of asset market participation, and Broer et al., 2020 and Walsh, 2017, who introduce heterogeneity by assuming one agent (the capitalist) holds all claims on profits but does not supply labor, while the other agent (the worker) has no claims on profits and supplies all labor.

Second, we analyze optimal monetary policy in response to productivity shocks within an economy with a distorted steady state. The study of optimal monetary policy goes back to Rotemberg and Woodford, 1997 who propose a method for deriving an optimal monetary policy that maximizes the utility of the representative household. Erceg et al., 2000 formulate optimal monetary policy under monopolistic competition and staggered nominal contracts. Clarida et al., 1999 derive optimal policy both with and without commitment within the standard New Keynesian model. Woodford, 2002 derives a social loss function from the welfare of underlying agents to study optimal monetary policy. Benigno and Woodford, 2005 show how to compute a valid quadratic approximation of the social welfare function even when the steady state is distorted.

Third, we combine the two aforementioned strands by analyzing how optimal monetary policy depends on the degree of income inequality in a TANK. A related literature analyzes how agent heterogeneity affects monetary policy in TANKs. Curdia and Woodford, 2010 study how monetary policy depends on the heterogeneity of preferences for consumption smoothing and dis-utility from working. Nisticò, 2016 and Bilbiie and Ragot, 2017 study how monetary policy depends on heterogeneous asset market participation.

Our paper also relates to papers on monetary policy within the class of Heterogenous Agent New Keynesian (HANK) models. ${ }^{3}$ In this environment, Kaplan et al., 2018 show that consumption responses to monetary policy actions are chiefly driven by the indirect income effect, rather than by the direct effect through inter-temporal substitution. They also show that this is not the case under a representative agent but also holds under a TANK. Gornemann et al., 2016 study how preferences for different monetary policy rules vary across agents in a HANK where the idiosyncratic unemployment risk depends on systematic monetary policy. There, the central bank operates a Taylor rule targeting unemployment and inflation. The median-wealth household favors a stronger central bank response to unemployment compared

\footnotetext{
${ }^{3}$ HANK models differ from TANK models by allowing the share of agents on their Euler equation to respond endogenously to shocks.
} 
to the wealthiest households because more unemployment stabilization provides consumption insurance. Bhandari et al., 2018; Acharya et al., 2020 study optimal monetary policy in a HANK where agents differ also in the ability to trade assets. In this setting, the central bank has an incentive to distribute the effect of the shock more evenly across agents and this incentive outweighs usual price stability considerations. Acharya et al., 2020 find similar results in a HANK economy with constant absolute risk aversion (CARA) preferences and normally distributed shocks allowing for analytical solutions.

Closest related to our work is Debortoli and Gali, 2017. They build a TANK model with price rigidities and two types of agents: Ricardians and Keynesians. Ricardian consumers have full access to bond and stock markets, while Keynesian consumers are "Hand-to-Mouth" consuming their current labor income at all times. In this setting, the central bank faces a non-trivial trade-off: it is not possible to simultaneously stabilize inflation, the output gap, and consumption inequality. We extend their analysis in three important dimensions: (i) we study optimal monetary policy when inequality is present in the steady state, a distortion that may have implications for optimal policy, and that is a feature consistent with the data; (ii) we consider a reduced form technological bias in wages to match the responses of consumption of different agents to productivity shocks in the U.S.; (iii) we study how inequality affects the use of Taylor rules. In addition, we study all three extensions under wage rigidities. All of these aim at, on the one hand, realistically capturing features of U.S. micro-data and, on the other hand, allowing the study of richer forms of inequality.

Organization. The remainder of the paper is organized as follows. In Section 2, we set up our model, which we then use in Section 3 to study whether the central bank should place a weight on inequality when conducting optimal monetary policy. In Section 4 we study whether introducing inequality in standard Taylor rules is welfare increasing. Section 5 concludes.

\section{A Stylized Model with Steady-state and Transitional Inequality}

This Section lays out the model we use to study the interactions between inequality and monetary policy in the presence of technology shocks. This model builds on Debortoli and Gali, 2017. The modeling of wealth inequality is limited to an uneven distribution of profits, while there are no aggregate savings in equilibrium.

The economy is populated by a continuum of infinitely lived households, indexed by $i \in[0,1]$. Households have the same preferences, and choosing consumption and savings to maximize utility. A fraction $\lambda$ of households - so called Keynesians - does not have access to financial markets, and hence their consumption is fully determined by their income, i.e. they are "hand-to-mouth". The remaining $1-\lambda$ fraction - Ricardians - have access to financial markets where they can trade bonds and stocks. Because all Ricardians are the same, there is no net supply of bonds, and Ricardians hold all equity in the economy - an extreme form of wealth inequality which translates into income inequality as profits are distributed as dividends. Households supply labor to intermediate goods producers and receive wage income in exchange. In

\footnotetext{
${ }^{4}$ De Giorgi and Gambetti, 2017 finds an uneven impact of TFP shocks on consumption. In particular, the right tail of the consumption distribution, comprised mostly of highly educated individuals, has a larger response than the poorer part of the distribution.
} 
addition, productivity shocks are assumed biased towards the wages of Ricardian agents, a reduced form skill-bias technological change.

The supply side features monopolistic competition among intermediate goods producers and a single representative firm that combines differentiated intermediate goods into a final consumption good. All households are subject to taxation in the form of i) lump sum transfers imposed to finance subsidies to intermediate goods producers to undo monopolistic distortions, and ii) redistribution policies based on dividend taxes.

\subsection{A Ricardian Agent, $r$}

A Ricardian agent, $r$, obtains utility from consumption $C_{r t}$, and disutility from labor $N_{t}$. She takes income $Y_{r t}$ as given and chooses consumption $C_{r t}$ and nominal bond holdings $B_{r t}$, with real value $b_{r t}=\frac{B_{r t}}{P_{t}}$, to solve

$$
\begin{aligned}
& \max E_{0} \sum_{t=0}^{\infty} \beta^{t}\left[\ln C_{r t}-\chi \frac{N_{t}^{1+\phi}}{1+\phi}\right] \\
& \text { s.t. } C_{r t}+b_{r t}=b_{r t-1} \frac{R_{t-1}}{\Pi_{t}}+Y_{r t}
\end{aligned}
$$

where $\beta$ is a preference discount factor, $R_{t}$ is the gross nominal rate on riskless bonds, $\Pi_{t}$ is gross inflation. Taking first order conditions yields the consumption Euler equation:

$$
C_{r t}^{-1}=\beta R_{t} E_{t}\left(C_{r t+1}^{-1} \frac{1}{\Pi_{t+1}}\right)
$$

In the background there is an implicit borrowing constraint that never binds.

The income of a Ricardian agent is composed of labor income, net-of-tax dividend payments, ${ }^{5}$ taxes, and transfers.

$$
Y_{r t}=\frac{1-\lambda\left(\frac{A_{t}}{\bar{A}}\right)^{-\gamma}}{1-\lambda} w_{t} N_{t}+\frac{1-\delta}{1-\lambda} d_{t}+t_{r t}-T_{p} Y_{t}
$$

The labor income of a Ricardian agent is proportional to aggregate labor income $w_{t} N_{t}{ }^{6}$ This proportionality allows for the possibility that TFP shocks affect the labor income of Ricardians and Keynesians differently, shifting resources from one type to the other depending on the value of $\gamma$. If $\gamma>0$, Ricardians see a larger share of labor income following a positive TFP shock. Dividends are distributed equally among Ricardian agents and taxed at a rate $\delta$. Taxes $T_{p} Y_{t}$ (which are raised from households and redistributed as sales subsidies to intermediate firms) eliminate the distortions from monopolistic competition. Transfers $t_{r t}$ are chosen by the fiscal authority for redistribution purposes and are financed by the taxes on divi-

\footnotetext{
${ }^{5}$ In principle, Ricardian agents are able to trade shares of the firms. This would add terms into the budget constraint (1). However, due to market clearing and the assumption that Keynesian agents do not have access to financial markets, the terms would simplify to the profits flow part $\frac{1-\delta}{1-\lambda} d_{t}$ that we reported. Furthermore, the possibility to trade shares, would give rise to a second Euler equation for stocks. This is again irrelevant in our setting as stock prices affect equilibrium conditions only through fluctuations in profits.

${ }^{6}$ The term $\frac{1-\lambda\left(\frac{A_{t}}{\bar{A}}\right)^{-\gamma}}{1-\lambda}$ can be obtained by imposing that all labor income must be distributed, given that we assume $\left(\frac{A_{t}}{\bar{A}}\right)^{-\gamma}$ is the share of total wage income that accrues to Keynesian agents.
} 
dends.

\subsection{A Keynesian Agent, $k$}

The problem faced by any Keynesian agent, $k$, is similar to that of Ricardian agents, except that the Keynesian agent is constrained by a binding borrowing constraint, i.e. $b_{k t}=0 .^{7}$

$$
\begin{aligned}
& \max E_{0} \sum_{t=0}^{\infty} \beta^{t}\left[\ln C_{k t}-\chi \frac{N_{t}^{1+\phi}}{1+\phi}\right] \\
& \text { s.t. } C_{k t}+b_{k t}=b_{k t-1} \frac{R_{t-1}}{\Pi_{t}}+Y_{k t}
\end{aligned}
$$

Given the binding borrowing constraint the Keynesian agent is effectively "hand-to-mouth", i.e. the relevant condition for determining her consumption is her budget constraint. The Keynesian agent's income is composed of labor income, taxes, and transfers:

$$
Y_{k t}=\left(\frac{A_{t}}{\bar{A}}\right)^{-\gamma} w_{t} N_{t}-T_{p} Y_{t}+t_{k t}
$$

Note that there is no dividend income, unlike the case of Ricardians, and that if $\gamma>0$, the share of labor income that Keynesians receive decreases following a positive TFP shock, a reduced-form way of introducing skill-biased wages.

\subsection{Labor Supply}

All workers are assumed to supply the same amount of labor as per the following aggregate rule. ${ }^{8}$

$$
w_{t}=\chi N_{t}^{\phi} Y_{t}
$$

where $w_{t}$ is the real wage, $\phi$ is the inverse Frisch elasticity, and $\chi$ is a parameter for labor disutility. We use this labor market structure to abstract from distortions introduced indirectly by income inequality, avoiding, for example, that type-dependent labor supply would itself be a source of inequality.

\subsection{Markets for Goods}

Final Good Producer The final goods market is assumed to be perfectly competitive, where a representative firm combines intermediate goods into a single final good. The production function is CES, as is the price index and the implied demand functions for intermediate inputs:

$$
Y_{t}=\left[\int_{0}^{1} y_{j t}^{\frac{\theta_{p}-1}{\theta_{p}}} d j\right]^{\frac{\theta_{p}}{\theta_{p}-1}}, \quad P_{t}=\left[\int_{0}^{1} p_{j t}^{1-\theta_{p}} d j\right]^{\frac{1}{1-\theta_{p}}}, \quad y_{j t}=\left(\frac{p_{j t}}{P_{t}}\right)^{-\theta_{p}} Y_{t} .
$$

\footnotetext{
${ }^{7}$ The fact that Keynesian agents hit their borrowing constraint, while Ricardian agents do not can be micro founded by different discount factors, with Keynesian agents being less patient than Ricardian ones. Some examples that make similar assumptions are Eggertsson and Krugman, 2012, and Benigno et al., 2020.

${ }^{8}$ This rule corresponds to the intratemporal optimality condition that would arise in a representative agent model, where $C_{i t}=Y_{t}$.
} 
Intermediate Goods Producers Each intermediate goods producer $j$ takes as given the demand function $y_{j t}=\left(\frac{p_{j t}}{P_{t}}\right)^{-\theta_{p}} Y_{t}$, as well as the nominal wage $W_{t}$, and the production function $y_{j t}=A_{t} L_{j t}^{1-\alpha}$, and maximizes profits subject to a quadratic price adjustment cost. Intermediate goods are subject to sales subsidies (at a rate $T_{p}$ ).

Intermediate goods producer $j$ faces the following problem at time $t$ :

$$
\begin{aligned}
\max _{p_{j t}} & \left(1+T_{p}\right) \frac{p_{j t}^{1-\theta_{p}}}{P_{t}^{1-\theta_{p}}} Y_{t}-\frac{w_{t}}{A_{t}^{\frac{1}{1-\alpha}}}\left(\frac{p_{j t}}{P_{t}}\right)^{\frac{-\theta_{p}}{1-\alpha}} Y_{t}^{\frac{1}{1-\alpha}}-\frac{\psi_{p}}{2} Y_{t}\left[\frac{p_{j t}}{p_{j t-1}}-1\right]^{2}+ \\
& +\beta\left(\frac{C_{r t+1}}{C_{r t}}\right)^{-1}\left\{\left(1+T_{p}\right) \frac{p_{j t+1}^{1-\theta_{p}}}{P_{t+1}^{1-\theta_{p}}} Y_{t+1}-\frac{w_{t+1}}{A_{t+1}^{\frac{1}{1-\alpha}}}\left(\frac{p_{j t+1}}{P_{t+1}}\right)^{\frac{-\theta_{p}}{1-\alpha}} Y_{t+1}^{\frac{1}{1-\alpha}}-\frac{\psi_{p}}{2} Y_{t+1}\left[\frac{p_{j t+1}}{p_{j t}}-1\right]^{2}\right\}
\end{aligned}
$$

Assuming symmetry across firms, or that $p_{j t}=P_{t}$, the first order condition boils down to the New Keynesian Phillips curve.

$$
\Pi_{t}\left[\Pi_{t}-1\right]=\beta\left(\frac{C_{r t+1}}{C_{r t}}\right)^{-1} \frac{Y_{t+1}}{Y_{t}} \Pi_{t+1}\left[\Pi_{t+1}-1\right]+\frac{\theta_{p}}{\psi_{p}}\left[\frac{1}{1-\alpha} \frac{w_{t}}{A_{t}^{\frac{1}{1-\alpha}}} Y_{t}^{\frac{\alpha}{1-\alpha}}-\left(1+T_{p}\right) \frac{\theta_{p}-1}{\theta_{p}}\right]
$$

Symmetry also leads to the following aggregate production function and an aggregate equation for real dividends.

$$
\begin{aligned}
Y_{t} & =A_{t} N_{t}^{1-\alpha} \\
d_{t} & =\left(1+T_{p}\right) Y_{t}-w_{t} N_{t}-\frac{\psi_{p}}{2} Y_{t}\left(\Pi_{t}-1\right)^{2}
\end{aligned}
$$

\subsection{Market Clearing}

Goods and bond markets clear: ${ }^{9}$

$$
\begin{aligned}
Y_{t} & =\lambda C_{k t}+(1-\lambda) C_{r t}+\frac{\psi_{p}}{2} Y_{t}\left(\Pi_{t}-1\right)^{2} \\
\int b_{i t} d i & =0
\end{aligned}
$$

\subsection{Fiscal Policy}

Fiscal policy has two independent parts. First, a sale subsidy, $T_{p}$, is given to intermediate producers, financed by lump sum taxes on all households. Second, profits are taxed at rate $\delta$ and then redistributed according to the following rules:

$$
\begin{aligned}
t_{k t} & =(1-\tau) \delta d_{t} \\
t_{r t} & =\frac{\delta d_{t}-\lambda t_{k t}}{1-\lambda} .
\end{aligned}
$$

\footnotetext{
${ }^{9}$ Note that we have already substituted the market clearing condition for labor $N_{t}=\int_{0}^{1} L_{j t} d j=L_{t}$.
} 
$1-\tau$ represents the extent to which taxed profits $\delta d_{t}$ are distributed to Keynesians. $\tau$ thus controls income inequality after taxes and transfers in steady-state, as well as over the business cycle. ${ }^{10}$ A higher value of $\tau$ thus means less (more) inequality, as less (more) profits is redistributed from Ricardians to Keynesians. The remaining taxed profits are then transferred to Ricardian agents per equation (13).

\subsection{Natural Output}

We define "natural output" as the output obtained if prices were flexible:

$$
Y_{t}^{n}=A_{t}\left[\left(1+T_{p}\right)(1-\alpha) \frac{\theta_{p}-1}{\theta_{p}} \frac{1}{\chi}\right]^{\frac{1-\alpha}{1+\phi}}
$$

\subsection{Equilibrium}

A competitive equilibrium in this economy is defined by two elements: 1) a sequence of stochastic processes $\left\{Y_{t}, Y_{t}^{n}, Y_{r t}, Y_{k t}, C_{r t}, C_{k t}, N_{t}, R_{t}, \Pi_{t}, w_{t}, d_{t}, t_{r t}, t_{k t}, b_{r t}, b_{k t}\right\}_{t=0}^{\infty}$; and 2) an exogenous process $\left\{A_{t}\right\}_{t=0}^{\infty}$, such that Ricardian agents solve their maximization problem respecting budget constraint (1) and consumption Euler equation (2); Keynesian agents respect their budget constraint (4); income flows are defined by (3) and (5) ; labor supply is defined by (6); output is produced according to the aggregate production function (8) and dividends are defined by (9); inflation follows the New Keynesian Phillips curve (7); transfers are defined by (12) and (13); goods market clears (10); the natural output levels is defined by (14); bond positions are such that $b_{k t}=\underline{b}=0$ and the bonds market clears (11). The model is closed by specifying monetary policy. Monetary policy affects the equilibrium through the Euler equation of the Ricardian agent and through general equilibrium effects on wages and profits. In the next sections we consider two possibilities: 1) a central bank that pursues welfare-based optimal monetary policy, and 2) a central bank that pursues different ad hoc interest rate rules.

\subsection{Approximate (Linearized) Equilibrium}

Following the tradition of the New Keynesian literature, we log-linearize the equilibrium conditions around the deterministic steady state. For a variable $x_{t}$, whose steady state value is $\bar{x}$, we define $\hat{x}_{t} \equiv \ln x_{t}-\ln \bar{x}$.

Log-linearized equilibrium conditions follow. We ignore the budget constraint of Ricardian agents due to Walras Law. Furthermore, we make use of the constant bond positions to disregard $b_{r t}, b_{k t}$, and to simplify $Y_{r t}=C_{r t}, Y_{k t}=C_{k t}$. An approximate equilibrium (here we report the Taylor rule for monetary policy) in this economy is made of two elements: 1) a sequence of stochastic processes $\left\{\hat{Y}_{t}, \hat{Y}_{t}^{n}, \hat{C}_{r t}, \hat{C}_{k t}, \hat{N}_{t}, \hat{R}_{t}\right.$,

\footnotetext{
${ }^{10}$ This is a departure from Debortoli and Gali, 2017's assumption of different taxes in the steady-state and over the business cycle.
} 
$\left.\hat{\Pi}_{t}, \hat{w}_{t}, \hat{d}_{t}, \hat{t}_{r t}, \hat{t}_{k t}\right\}_{t=0}^{\infty} ;$ and 2) an exogenous process $\left\{\hat{A}_{t}\right\}_{t=0}^{\infty}$, such that:

$$
\begin{aligned}
& \hat{C}_{r t}=E_{t} \hat{C}_{r t+1}-\hat{R}_{t}+E_{t} \hat{\Pi}_{t+1} \\
& \frac{\bar{C}_{k}}{\bar{w} \bar{N}} \hat{C}_{k t}+\frac{T_{p} \bar{Y}}{\bar{w} \bar{N}} \hat{Y}_{t}-\frac{\bar{t}_{k}}{\bar{w} \bar{N}} \hat{t}_{k t}=-\gamma \hat{A}_{t}+\hat{w}_{t}+\hat{N}_{t} \\
& \hat{w}_{t}=\phi \hat{N}_{t}+\hat{Y}_{t} \\
& \hat{Y}_{t}=\hat{A}_{t}+(1-\alpha) \hat{N}_{t} \\
& \left(1+T_{p}\right) \hat{Y}_{t}=\frac{\bar{d}}{\bar{Y}} \hat{d}_{t}+\frac{\bar{w} \bar{N}}{\bar{Y}}\left(\hat{w}_{t}+\hat{N}_{t}\right) \\
& \hat{\Pi}_{t}=\beta E_{t} \hat{\Pi}_{t+1}+\frac{\theta_{p}}{\psi_{p}}\left(\hat{w}_{t}+\frac{\alpha}{1-\alpha} \hat{Y}_{t}-\frac{1}{1-\alpha} \hat{A}_{t}\right) \\
& \hat{d}_{t}=\frac{(1-\lambda) \bar{t}_{r}}{\delta \bar{d}} \hat{t}_{r t}+\frac{\lambda \bar{t}_{k}}{\delta \bar{d}} \hat{t}_{k t} \\
& \hat{t}_{k t}=\hat{d}_{t} \\
& \hat{Y}_{t}=\lambda \frac{\bar{C}_{k}}{\bar{Y}} \hat{C}_{k t}+(1-\lambda) \frac{\bar{C}_{r}}{\bar{Y}} \hat{C}_{r t} \\
& \hat{Y}_{t}^{n}=\hat{A}_{t} \\
& \hat{R}_{t}=\phi_{\pi} \hat{\Pi}_{t}+\phi_{y} \hat{Y}_{t}
\end{aligned}
$$

\subsection{Steady State}

The steady state of this economy generally depends on structural parameters that i) concern monopolistic distortions $\left(T_{p}\right.$ and $\left.\theta_{p}\right),{ }^{11}$ ii) govern fiscal redistribution $(\delta, \tau)$, iii) characterize the production function $(\alpha, \bar{A})$, iv) define preferences $(\beta, \phi)$, and v) characterize population shares $(\lambda)$. Note that the steady state does not depend on $\gamma$ as it governs only the degree of "cyclical" skill-biasedeness.

\subsection{Exogenous Process}

Throughout the paper we consider the economy to start in the steady state and a one time, unexpected shock $\epsilon_{t}$ that increases productivity $A_{t}$ at $t=0$. Specifically we assume that

$$
A_{t}=\bar{A} e^{\epsilon_{t}}
$$

which is linearized to

$$
\hat{A}_{t}=\epsilon_{t} .
$$

We assume that $\epsilon_{t}=\rho \epsilon_{t-1}$, and that $\epsilon_{0}=0.01$. The evolution of the shock is deterministic.

\footnotetext{
${ }^{11}$ We assume that $\left(1+T_{p}\right) \frac{\theta_{p}-1}{\theta_{p}}=1$.
} 


\subsection{Calibration}

The main novel parameters to be calibrated are $\lambda, \tau$, and $\gamma$. The rest are set to standard values found in the literature.

We set $\lambda=0.4$, which is in the middle of a wide range used in the literature, see Coenen et al., 2012. We calibrate $\tau$ to 0.93 to match the ratio of non-labor income of households in the top 60 percent of the income distribution to the bottom 40 percent in the Survey of Consumer Finances for the U.S. in 2016. ${ }^{12}$

$\gamma$ is calibrated to match the effect of a productivity shock on consumption across the income distribution as estimated in De Giorgi and Gambetti, 2017. This is done assuming that monetary policy follows a Taylor rule with standard response parameters. ${ }^{13}$

Table 1 summarizes the calibration used henceforth.

Table 1: Calibration

\begin{tabular}{c|c|c}
\hline \hline Parameter & Value & Note \\
\hline$\lambda$ & 0.4 & Share of Keynesian agents \\
$\tau$ & 0.93 & Redistribution of Profits \\
$\delta$ & 1 & Redistribution of Profits \\
$\gamma$ & 1.67 & Degree of Skill Bias \\
$\alpha$ & 0.25 & Profits Share \\
$\beta$ & 0.9925 & Discount Factor \\
$\chi$ & 1 & Labor Disutility \\
$\phi$ & 1 & Inverse Frisch Elasticity \\
$\theta_{p}$ & 9 & CES Elasticity \\
$\psi_{p}$ & 372.8 & Price Adjustment \\
$\rho$ & 0.9 & Persistence of Shock \\
\hline
\end{tabular}

\section{Optimal Monetary Policy}

In this section, we show that a welfare-maximizing central bank caring equally about all agents in the economy described in Section 2 should place a non-zero weight on stabilizing consumption inequality, beyond the usual objectives of stabilizing inflation and output gaps. However, the weight on consumption inequality under such an optimal policy is relatively small, and those on inflation and output gaps are only marginally affected by the presence of inequality. This means that a policy where the central bank maximizes the welfare of the average agent, ignoring actual inequality in the economy, only entails small welfare losses compared to the fully optimal policy that incorporates inequality. These results are robust to the inclusion of wage rigidites, see Appendix Section A.4. In what follows, we derive the social welfare function and study the resulting optimal monetary policy.

\footnotetext{
${ }^{12}$ This ratio is 24.5. $\tau$ is chosen so that $\frac{\bar{t}_{r}}{\bar{t}_{k}}=24.5$. Notice that we have also imposed $\delta=1$ meaning the dividend tax base is the total amount of dividends.

${ }^{13}$ We use $\phi_{\pi}=1.5$ and $\phi_{y}=0.125$ in equation 25 .
} 


\subsection{The Social Welfare Function}

Consider a central bank that aims to maximize the welfare of all agents in the economy described in Section 2 and that values each agent equally. ${ }^{14}$ The welfare function can be written as:

$$
\mathbf{W}=E_{0} \sum_{t=0}^{\infty} \beta^{t}\left[\lambda \ln C_{k t}+(1-\lambda) \ln C_{r t}-\frac{N_{t}^{1+\phi}}{1+\phi}\right]
$$

Note that the consumption portion of utility is weighted by each type's share in the population, with Keynesians accounting for $\lambda$ and Ricardians for $1-\lambda$, while the separable leisure portion is not weighted because of the assumed aggregate labor supply decision in equation (6).

The welfare function in (26) can be re-written as a quadratic in three gaps: ${ }^{15}$

$$
\mathbf{W} \approx E_{0} \sum_{t=0}^{\infty} \beta^{t}\left\{-\frac{1}{2} W_{\Pi} \hat{\Pi}_{t}^{2}-\frac{1}{2} W_{Y}\left(\hat{Y}_{t}-\hat{Y}_{t}^{*}\right)^{2}-\frac{1}{2} W_{\Delta_{c}} \hat{\Delta}_{c t}^{2}\right\}+T_{0}+\text { t.i.p. }
$$

where $\hat{\Pi}_{t}$ is the deviation of inflation from its steady state value, or the inflation gap, $\hat{Y}_{t}-\hat{Y}_{t}^{*}$ is the deviation of output from its welfare optimizing level, or what we henceforth call output gap, ${ }^{16}$ and $\hat{\Delta}_{c t}=$ $\hat{C}_{r t}-\hat{C}_{k t}$ is the change in the relative consumption of the Ricardian and the Keynesian agent, or the consumption inequality gap, all at time $t$. A positive $\hat{\Delta}_{c}$ reflects higher consumption inequality since the Ricardian agent has higher steady-state consumption to begin with, provided $\tau>0 . T_{0}$ is a predetermined quantity that depends only on gaps at time zero. t.i.p. stands for terms independent of (monetary) policy. Appendix A.1 contains the detailed derivations and the analytical expression for each weight and for $T_{0}$.

\subsection{Optimal Policy}

Figure 1 presents the weights on the three gaps in equation (27) depending on the degree to which profits are taxed and redistributed, as governed by $\tau .{ }^{17}$ Two things are apparent: (i) the weight on consumption inequality is not zero but it is very small compared to that on the output gap, which itself is much smaller than that placed on the inflation gap (the weights in our baseline calibration are $0.2,5$ and 310 , respectively); (ii) the weight on inflation gap is indeed the largest but declines with larger $\tau$, with the opposite being true for the weight on output gap (the weight on inflation gap declines by 17 percent from the egalitarian society, $\tau=0$, relative to that in our baseline calibration, while the weight on output gap rises by 79 percent across the two calibrations).

\footnotetext{
${ }^{14}$ This follows the optimal policy literature, see e.g. Woodford, 2002.

${ }^{15}$ Simplifying (26) into a quadratic loss function involves two steps. First, we take a second-order expansion following Rotemberg and Woodford, 1997 and Woodford, 2002. In many applications, such a second-order expansion leads immediately to a simple quadratic loss function because the steady state is efficient. For example, this is the case in Debortoli and Gali, 2017, due to $\tau$ being zero. In our economy though, the steady-state is inefficient and thus the second-order expansion of equation (26) includes linear terms on crucially the output gap. To deal with the latter, we adapt the method in Benigno and Woodford, 2005 and derive the objective function under "timeless commitment", in which the loss function is quadratic up to a term that is set at time zero.

${ }^{16}$ This definition of output gap is not standard because here $\hat{Y}_{t}^{*}=W_{A Y} \hat{A}_{t}$, as opposed to just $\hat{A}_{t}$, as in the representative agent model. $W_{A Y} \neq 1$ arises due to tech-bias in wages, see Appendix A.1 for details.

${ }^{17}$ Welfare weights do not depend on $\gamma$, the degree of skill-bias in wages, because the weights themselves are functions evaluated in the steady state and skill bias is immaterial in the steady state. However, $\gamma$ affects welfare directly because it changes the welfare relevant measure of potential output and indirectly through its impact on the dynamics of the economy. See Appendix A.1 for the analytical expressions.
} 
Figure 1: Welfare Weights and Redistribution Parameter $\tau$

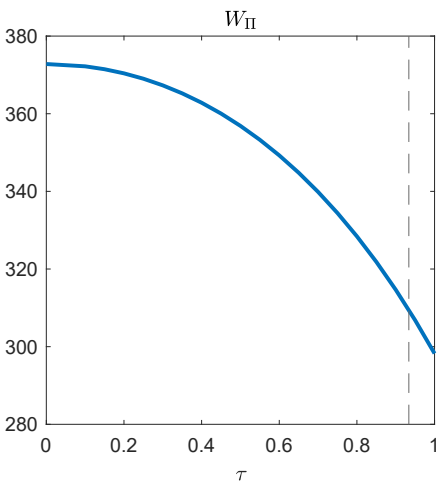

(a) Weight on Inflation Gap

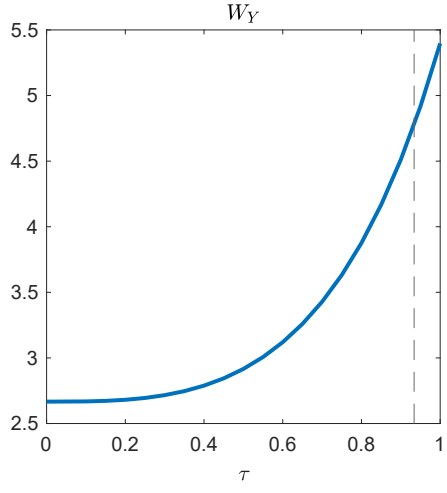

(b) Weight on Output Gap

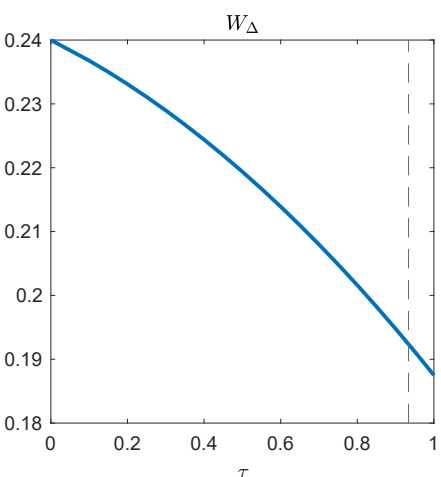

(c) Weight on Consumption Inequality Gap

Notes: The panels show the three weights in equation (27) as a function of the extent to which dividends are redistributed, governed by $\tau$. All other parameters are set according to the baseline calibration in Table 1 .

The weight on consumption inequality is small because aggregate fluctuations affect all agents, while fluctuations in consumption inequality are second-order and disproportionately affect one type of agent. This weight depends crucially on the level of steady-state inequality, as governed by $\tau$, and the fraction of Keynesian agents, $\lambda$. In our calibration, $\lambda$ is smaller than one half, and thus Figure 1 shows that the weight on consumption inequality declines with $\tau$. In an economy where Keynesians form a small group, transferring resources to them is less appealing to the social planner and is even less appealing when $\tau$ is higher as redistribution involves taking from the majority to more than proportionately benefit the minority. Finally we note that looking at the weight alone does not completely relay the possible welfare loss accruing to different types of gaps because the gaps themselves vary in size after a shock.

Secondly, why would the central bank care progressively less about inflation and more about the output gap the higher the $\tau$, and hence the lower the redistribution in this economy? The cost of any inflation gaps is directly borne by firms due to Rotemberg pricing, reducing their profits. When $\tau$ is large, such profits accrue principally to the already better-off Ricardian agent. Thus, a central bank that values agents equally will on the margin more easily tolerate an inflation gap when $\tau$ is high because that ends up being re-distributive and small gains for the poor agent when inequality is high are particularly welfare-enhancing. At the same time, an economy that is deeply unequal due to large $\tau$ is one where the Keynesian agent will overwhelmingly depend on wages for her income. Stabilizing the labor supply, and thus output, becomes relatively more important in such an unequal economy.

The policy that maximizes (27) subject to a first order approximation of the Philips curve, the resource constraint (equations 20 and 23) and a given level of $T_{0}$ is henceforth denoted as optimal policy. ${ }^{18}$

\subsection{RANK-Optimal Policy - A Policy For the Average Agent}

In order to assess the benefits of pursuing the optimal policy explained above, we define an alternative "RANK-optimal" policy in which the central bank maximizes welfare as if the economy has a representative agent, thus ignoring the existence of inequality. In other words, RANK-optimal policy focuses on

\footnotetext{
${ }^{18}$ See Appendix A.2 for details.
} 
maximizing the average consumption in the economy rather than internalizing the differences in consumption across the two agents.

Under the assumption that the central bank ignores inequality, its objective function simplifies from (27) to: ${ }^{19}$

$$
\mathbf{W}_{\mathbf{R A N K}} \approx \sum_{t=0}^{\infty} \beta^{t}\left\{-\frac{1}{2} W_{R A N K, \Pi} \hat{\Pi}_{t}^{2}-\frac{1}{2} W_{R A N K, Y}\left(\hat{Y}_{t}-\hat{A}_{t}\right)^{2}\right\}+T_{0}+\text { t.i.p. }
$$

Weights, $W_{R A N K, \Pi}, W_{R A N K, Y}$ coincide with those presented in Figure 1 when $\tau=0$. They are the standard weights in the RANK literature, e.g. see Galí, 2015. $T_{0}$ is constrained to be the same value attained by optimal policy.

\subsection{Responses to TFP shocks}

Figure 2: Impulse Response to a Positive TFP shock: Optimal vs RANK-Optimal Policy

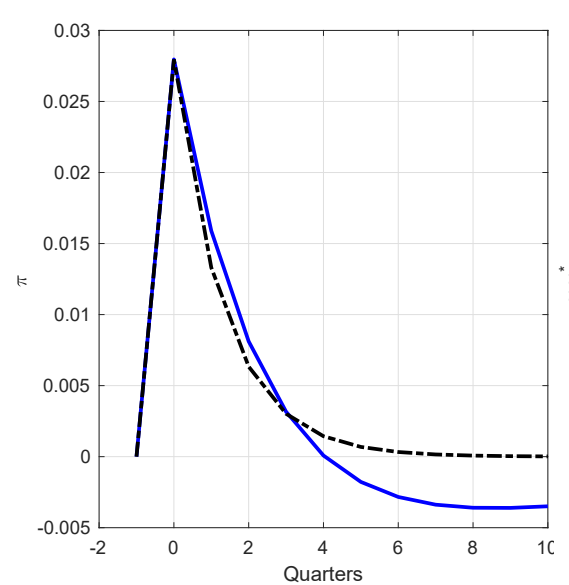

(a) Inflation Gap

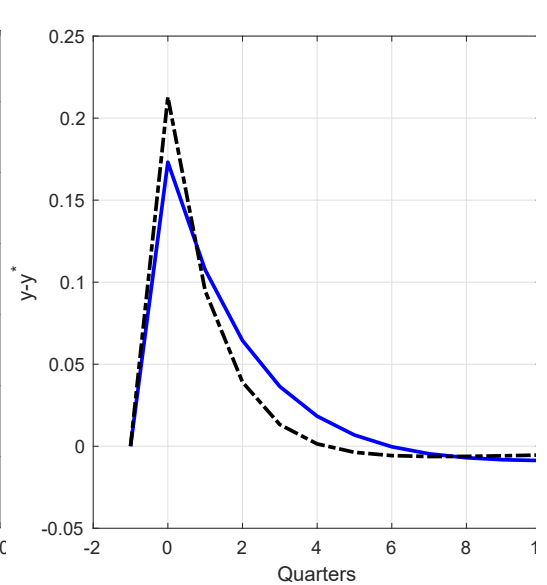

(b) Output Gap

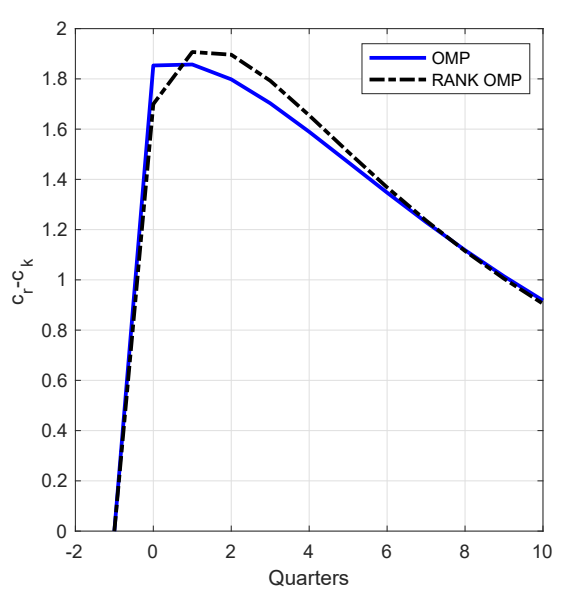

(c) Consumption Inequality Gap

Notes: The three panels show the impulse response functions of inflation, output and consumption inequality gaps, respectively, under the fully optimal policy (blue) and the RANK-optimal policy (black dash-dotted). The y-axis measures the deviation from each variable's steady state, measured in percentage points.

Figure 2 shows impulse responses of key gaps to a positive TFP shock comparing optimal and RANKoptimal policies.

Optimal policy cushions the rise in consumption inequality (third panel) more forcefully than RANKoptimal policy. In doing so, optimal policy allows for slightly larger inflation and output gaps on average (first and second panels, respectively), reflecting its full internalization of the trade-off between stabilizing aggregates and consumption inequality. ${ }^{20}$

However, the impulse responses of optimal and RANK-optimal policies are nearly identical and thus optimal policy is only marginally better than RANK-optimal policy. Figure 3 shows the welfare loss in consumption-equivalent terms, ${ }^{21}$ using (27) under each of these 2 policies, depending on $\tau$ and $\gamma$ and de-

\footnotetext{
${ }^{19}$ See Appendix A.3 for details.

${ }^{20}$ If wages are also rigid, we show that these insights remain unchanged, see Appendix Figure A2.

${ }^{21}$ We compute such compensation in percent of steady-state consumption of the average agent, as in Ravenna and Walsh, 2011. Specifically, we compute $x$ as the consumption equivalent representing the permanent increase in consumption that
} 
composing losses into three sources stemming from each of the three gaps being non-zero. Under our calibration, the welfare gain is only about $2.5 \times 10^{-6}$ percent in yearly consumption-equivalent terms. In relative terms, this means that moving to fully optimal policy only increases welfare by 1 percent. ${ }^{22}$

Unsurprisingly, optimal policy achieves higher welfare overwhelmingly through reduced consumption inequality (yellow area being the largest and positive) at the expense of larger losses due to inflation and to some extent output gaps, although the latter depends crucially on $\tau$ but not $\gamma$. In our baseline calibration, optimal policy delivers higher welfare through a smaller output gap reflecting a larger focus of the social planner on the output gap when profits are not much distributed or when the skill-bias is more pronounced ( $\tau$ or $\gamma$ are high).

Figure 3: Welfare of Optimal vs. RANK-Optimal Policy in Consumption Equivalent Terms
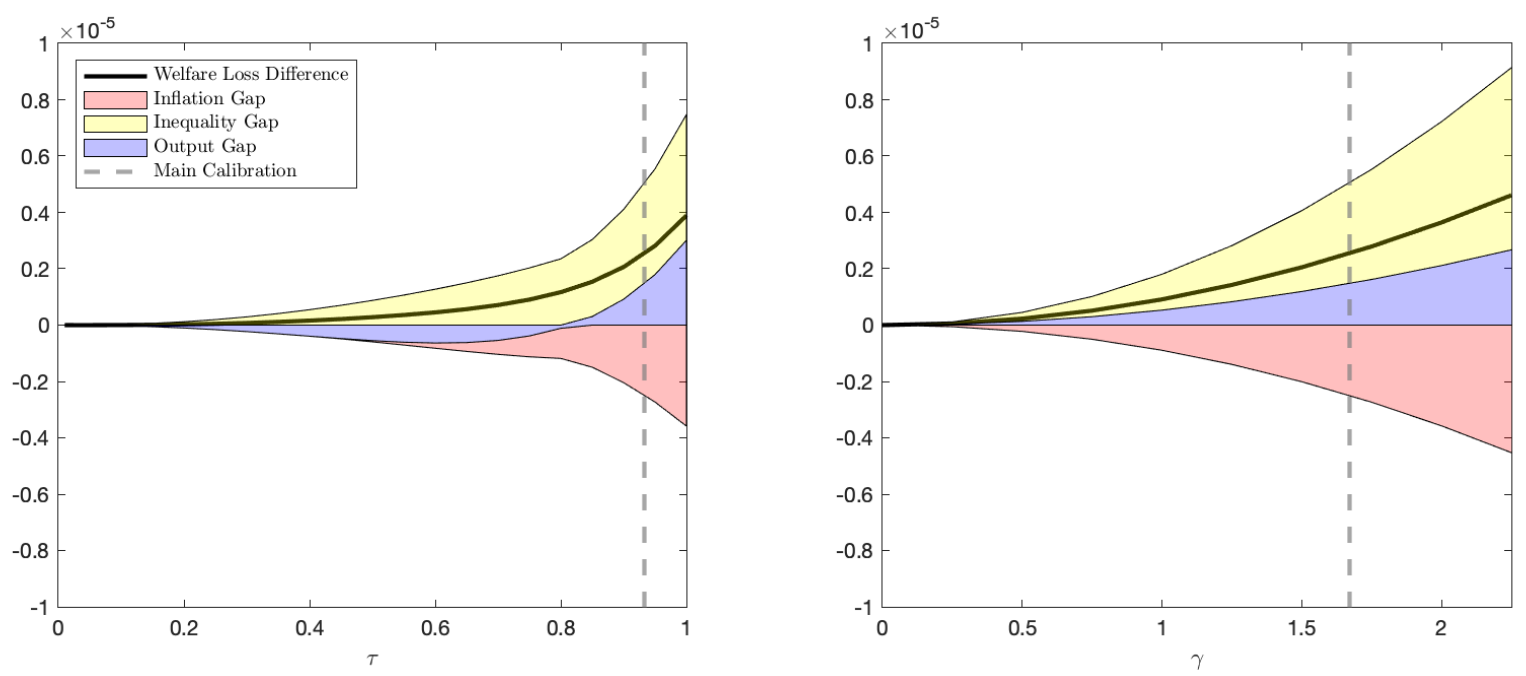

Notes: The panels show how welfare under optimal policy and RANK-optimal policy compare depending on parameters that control inequality. The black line shows the overall gain, in consumption-equivalent terms of moving from RANK-optimal to fully optimal monetary policy. The colored areas decompose the overall welfare gain in terms of the three gaps (inflation, output, and consumption inequality gaps). The left (right) panel shows how this welfare comparison depends on $\tau$ ( $\gamma$ ), shown in the x-axis. The vertical dashed line denotes our baseline calibration.

\section{Augmented Taylor rules}

Optimal monetary policy is hard to implement in practice. First, it requires extensive information about the structure of the economy including the paths of all variables under different scenarios. Second, it may not be credible to pre-commit to the full paths of all three gaps. For this reason policy makers sometimes make reference to simpler monetary policy rules. ${ }^{23}$ A famous example of such a rule is the one proposed by Taylor, 1993.

In this section, we study more practical "Taylor Rules". We show that central banks using such rules should set lower rates than they would otherwise because of inequality in response to a positive TFP shock.

would increase welfare by the amount considered. Consider two monetary policies, $a$ and $b$, with the former being welfaresuperior to the latter (i.e. $\left.W_{a}>W_{b}\right)$. We compute $x$ from the following formula $x=\exp \left[(1-\beta)\left(W_{a}-W_{b}\right)\right]-1$.

${ }^{22}$ The same qualitative results hold under wage rigidites, see Appendix Figure A3

${ }^{23}$ See for example https://www.federalreserve.gov/monetarypolicy/policy-rules-and-how-policymakers-use-them.htm and https://www.brookings.edu/blog/ben-bernanke/2015/04/28/the-taylor-rule-a-benchmark-for-monetary-policy/ 
The mechanism is simple: a policy of lower interest rates leads to higher wages on the margin, and thereby benefits disproportionately the poor, who rely more on labor income. Such a policy is beneficial not only because it lowers inequality, but also because it improves inflation and growth outcomes by avoiding an excessive tightening of the interest rate in response to a positive productivity shock. The same exact conclusions apply in a modified version of our model with wage rigidites, see Appendix Section A.4.

In the following two subsections, we discuss Taylor rules in two stages. Firstly, we evaluate the implications of the existence of inequality for standard Taylor rules without considering explicit inequality targeting. Secondly, we assess whether standard rules should indeed feature an explicit targeting of consumption inequality.

\subsection{Standard Taylor Rules and Inequality}

We evaluate Taylor rules of the following form:

$$
\hat{R}_{t}=\phi_{\pi} \hat{\Pi}_{t}+\phi_{y} \hat{Y}_{t}+\phi_{c}\left(\hat{C}_{r t}-\hat{C}_{k t}\right)
$$

Here $\hat{Y}_{t}$ is the deviation of output from steady state, ${ }^{24} \hat{\Pi}_{t}$ is the deviation of inflation from target, while $\hat{C}_{r t}-\hat{C}_{k t}$ measures inequality through the difference in consumption between workers and capitalists.

Table 2 compares different Taylor rules of the form defined in equation (28). It begins by evaluating a typical rule with $\phi_{\pi}=1.5$ and $\phi_{y}=0.125$ as in Galí, 2015, Chapter 3. It then contrasts such a rule with one where the output coefficient is zero or one where it is 1 . Finally, a rule with a high coefficient on inflation alone is also assessed. In this subsection, we keep $\phi_{c}=0$.

Table 2: Welfare in Taylor Rules - Standard Parametrization

\begin{tabular}{c|c|c|c|c|c|c|c|c} 
& $(1)$ & $(2)$ & $(3)$ & $(4)$ & $(5)$ & $(6)$ & $(7)$ & $(8)$ \\
\hline \hline$\tau$ & 0 & 0 & 0 & 0 & 0.93 & 0.93 & 0.93 & 0.93 \\
$\gamma$ & 0 & 0 & 0 & 0 & 1.67 & 1.67 & 1.67 & 1.67 \\
$\phi_{\pi}$ & 1.5 & 1.5 & 5 & 1.5 & 1.5 & 1.5 & 5 & 1.5 \\
$\phi_{y}$ & 0.125 & 0 & 0 & 1 & 0.125 & 0 & 0 & 1 \\
\hline Cons. Equiv. Loss & 0.4 & 0.1 & 0 & 1.4 & 2.7 & 1.9 & 0.3 & 4.6 \\
Cons. Equiv. $W-T_{0}$ & 0.5 & 0.2 & 0 & 1.9 & 1 & 0.6 & 0.1 & 2.1 \\
Inflation & 98.2 & 98.2 & 98.1 & 98.2 & 87.1 & 83.6 & 24 & 90.1 \\
Output & 1.8 & 1.8 & 1.9 & 1.8 & 3.4 & 3.2 & 1.5 & 3.7 \\
Inequality & 0 & 0 & 0 & 0 & 9.6 & 13.2 & 74.5 & 6.3 \\
\hline \hline
\end{tabular}

Notes: The table reports the consumption equivalent welfare loss, multiplied by 10000 (e.g. 1 means 0.0001 percent of steady state consumption); the dynamically relevant loss $\left(W-T_{0}\right)$ also in consumption equivalent terms; the shares of $W-T_{0}$ accounted by a non-zero inflation, output and inequality gaps. These shares may not sum to 100 due to rounding.

In an economy without inequality (columns (1)-(4), when $\tau=\gamma=0$ ) three conclusions emerge: (1) Taylor rules with zero weight on output fare better; (2) a large weight on inflation fares even better; (3) any welfare losses are disproportionately due to non-zero inflation gaps. These are all well understood in the literature (see for example Galí, 2015, Chapter 3) and reflect the disproportionately large weight on

\footnotetext{
${ }^{24}$ An alternative is to target output gaps, or the deviation of output from its equilibrium under flexible prices. As the purpose of this section is to investigate practical policy rules, we opt for the deviation from steady state which arguably is easier to measure. In the version of the Taylor rule where output, and not output gap, is targeted, there is an additional benefit to targeting consumption inequality because doing so allows to better proxy for the interest rate that would best respond to the output gap.
} 
inflation in the social welfare function of equation (27) and the complementarity between reducing inflation and output gaps under TFP shocks. This finding is often referred to as the "divine coincidence" by Blanchard and Galí, 2007.

When inequality is introduced in columns (5)-(8), the same three observations above continue to hold. Importantly, a higher parameter on inflation reduces total welfare losses from all three gaps. ${ }^{25}$ Note, that the welfare loss due to the consumption inequality gap can be sizeable, always larger than that of output gap and even larger than that stemming from inflation gaps when the Taylor rule reflects a strong inflation mandate (column 7).

Table 2 suggests that aggressively responding to inflation gaps is a superior policy. ${ }^{26}$ But the question remains on whether welfare can be improved by allowing a small weight on the consumption inequality gap $\left(\phi_{c} \neq 0\right.$ in equation (28)), even under the Taylor rule used in column 7 .

\subsection{Consumption inequality targeting}

Figure 4 shows the welfare achieved by the four rules studied in Table 2 when varying the parameter on the consumption inequality gap (or $\left.\phi_{c}\right) .{ }^{27}$ When $\tau$ and $\gamma$ are not zero, the standard Taylor rules in Table 2 achieve a higher welfare when $\phi_{c}$ becomes negative, or towards the left of each subplot. This is true across all four rules, with the maximum achieved around $\phi_{c} \in[-0.2,-0.05]$ except for the rule with an output weight of 1 , in which case it is preferable to use a much more negative $\phi_{c} \cdot{ }^{28}$

To understand better why it is useful to target consumption inequality, Figure 5 compares impulse responses of three gaps for the most standard Taylor rule (using $\phi_{\pi}=1.5$ and $\phi_{y}=0.125$ ). This is done with and without augmentation as well as those implied by the Optimal Monetary Policy explored in Section 3. For the augmented Taylor rule, the parameter on the consumption inequality gap is chosen to maximize welfare as defined in equation (27). Moreover, the same qualitative conclusions carry through if we had chosen any other of the three rules explored in Table 2.

Targeting the consumption inequality gap is welfare increasing. The consumption equivalent gain of moving to such an augmented Taylor rule is $7 \times 10^{-3}$ percent. This gain is large at about 96 percent of the loss under the standard Taylor rule as reported in Table 2. By having a negative coefficient on consumption inequality, the augmented Taylor rule delivers both lower consumption inequality but also lower absolute inflation and output gaps. Under the standard Taylor rule, a positive TFP shock leads to higher inequality and creates negative output and inflation gaps. By moving to a Taylor rule augmented with a negative parameter on the consumption inequality gap, interest rates are set lower than they would be otherwise following the same positive TFP shock. This increases wages and stimulates demand, thereby allowing to close inflation and output gaps. Higher wages also disproportionately help the poor and thus diminish the increase in inequality. Interestingly, the augmented Taylor rule with an optimized parameter on inequality goes beyond closing the negative inflation and output gaps and actually results in small positive gaps. Such a Taylor rule is essentially trading-off complete stabilization of macro gaps with stabilizing

\footnotetext{
${ }^{25}$ This can be seen by comparing the levels of consumption equivalent losses across different Taylor rules. For instance, consider the welfare loss from inflation under column 5 , which is $1.9 \times 0.982$. This is larger than the welfare loss from inflation under columns 7 , which is $0.6 \times 0.24$.

${ }^{26}$ The same broad patterns carry through to the case with wage rigidities, see Appendix Table A1.

${ }^{27}$ In this section, we ignore $T_{0}$ when calculating welfare. Including $T_{0}$ would not change the qualitative results and in fact welfare gains are even larger when it is taken into account. The disadvantage, though, is that welfare gains become harder to interpret, because welfare losses can become negative as the inflation gap enters $T_{0}$ linearly.

${ }^{28}$ Under wage rigidity, the same patterns are remarkably similar, see Appendix Figure A4.
} 


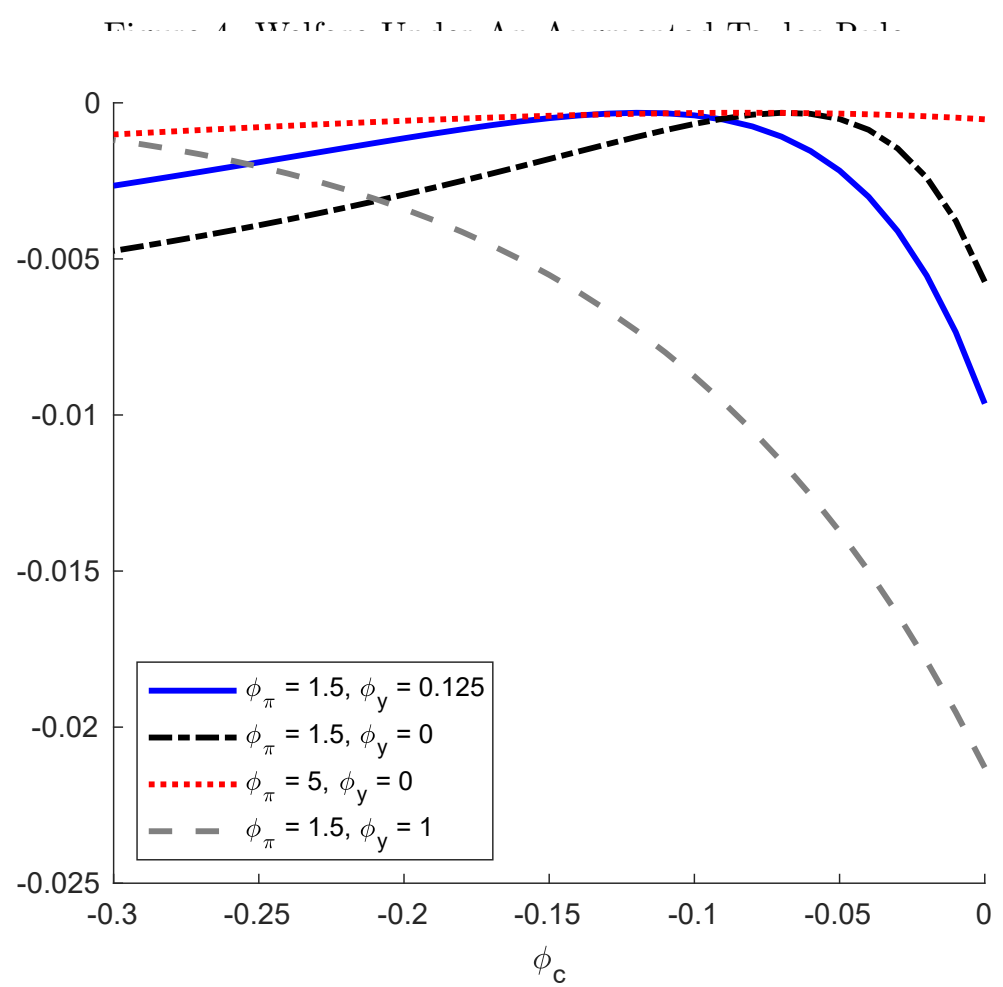

Notes: The figure shows how the part of welfare that is quadratic on gaps depends on the parameter on consumption inequality, $\phi_{c}$. Each line corresponds to the four Taylor rules evaluated in Table 2, columns (5)-(8), but in which we allow $\phi_{c}$ to be non-zero.

Figure 5: Impulse Response Functions: Standard and Augmented Taylor rules

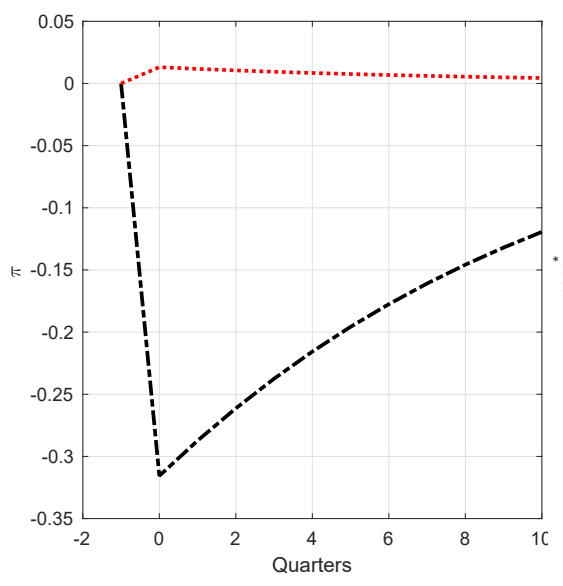

(a) Inflation Gap

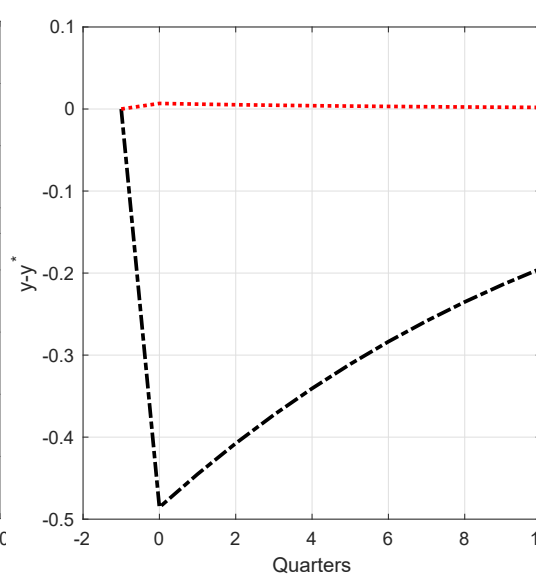

(b) Output Gap

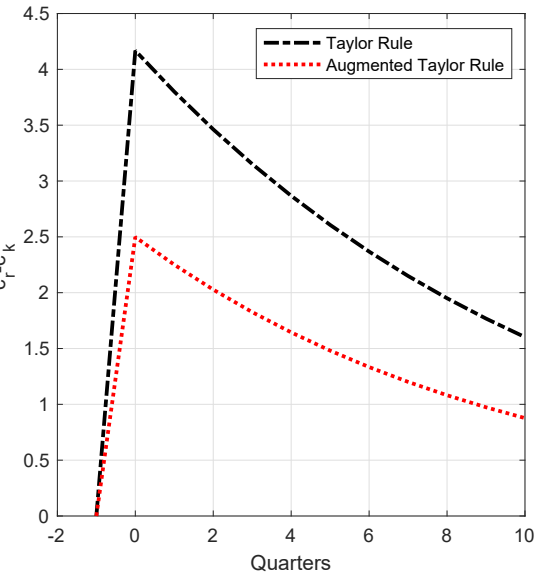

(c) Consumption Inequality Gap

Notes: The three panels show the Impulse Response Function of, respectively, inflation, welfare relevant output gap, and consumption inequality gap. The two lines correspond to two different policies under our baseline calibration. The black dashed-dotted line corresponds to the Standard Taylor rule using $\phi_{\pi}=1.5, \phi_{y}=0.125$ and $\phi_{c}=0$ and the red dotted line corresponds to the Augmented Taylor Rule where $\phi_{\pi}=1.5, \phi_{y}=0.125, \phi_{c}=\phi_{c}^{*}$. 
the consumption inequality gap. ${ }^{29}$

${ }^{29}$ The same applies to the case of rigid wages, see Appendix Figure A5. 


\section{Conclusion}

Should inequality factor into central banks' decisions? We analyzed this question using a stylized TwoAgent New Keynesian Model. We studied both optimal monetary policy whereby the central bank cares equally about all agents in the economy and Taylor rules whereby the central bank sets the interest rate based on the state of the aggregate economy.

Targeting consumption inequality does not improve welfare much if the central bank already implements optimal monetary policy ignoring inequality. A central bank should place a non-zero optimal social weight on the consumption inequality gap. However, the welfare gain of taking inequality explicitly into account is only about 1 percent of the loss under optimal monetary policy targeted to the average agent.

On the other hand, if the central bank implements monetary policy through a standard Taylor rule, then augmenting it with an inequality target can lead to higher welfare. We found that beyond targeting inflation and output gaps, the central bank can achieve higher welfare if placing a small negative weight on consumption inequality. This means that following a positive TFP shock that increases consumption inequality, the central bank should on the margin loosen its policy rate. Such an augmented Taylor rule would generate a gain equal to 96 percent of the loss under a standard Taylor rule, a gain much larger than that under optimal policy. Such an augmented Taylor rule allows both a more effective stabilization of consumption inequality but also stabilizes inflation and output gaps. Interestingly, our results do not depend on the existence of unemployment and how monetary policy can mitigate its volatility, which could be another motive to make monetary policy inequality-sensitive.

These conclusions are of course model specific. Although we believe the model captures important dimensions of inequality, it will be important for future research to assess whether these findings can be generalized. First, our analysis focused on an economy exposed to TFP shocks. It would be useful to extend the analysis to a broader set of shocks, including those on the demand side. Second, we modeled steadystate inequality arising from an unequal distribution of equity holdings and hence profits in an economy with a simplistic wealth distribution and no aggregate savings. This could be extended to inequality from other income sources, in particular from differentiated labor, and a richer form of wealth inequality, capturing differentiated savings and also holdings of illiquid assets like housing. The movements in other asset prices in response to monetary policy could also be modelled beyond stock prices. Finally, we restricted our analysis to monetary policy. It would be important to study how the conclusions above change when monetary policy is used in coordination with other policy tools. 


\section{References}

Acharya, Sushant, Edouard Challe, and Keshav Dogra (2020). "Optimal Monetary Policy According to HANK". Federal Reserve Bank of New York Staff Reports 916.

Benigno, Pierpaolo, Gauti B. Eggertsson, and Federica Romei (Apr. 2020). "Dynamic Debt Deleveraging and Optimal Monetary Policy". American Economic Journal: Macroeconomics 12.2, pp. 310-50. DOI: 10.1257/mac. 20160124. URL: https://www . aeaweb.org/articles?id=10.1257/mac . 20160124.

Benigno, Pierpaolo and Michael Woodford (2005). "Inflation stabilization and welfare: The case of a distorted steady state". Journal of the European Economic Association 3.6, pp. 1185-1236.

Bhandari, Anmol, David Evans, Mikhail Golosov, and Thomas Sargent (2018). "Inequality, Business Cycles and Monetary-Fiscal Policy". National Bureau of Economic Research Working Paper 24710.

Bilbiie, Florin O (2008). "Limited asset markets participation, monetary policy and (inverted) aggregate demand logic". Journal of economic theory 140.1, pp. 162-196.

Bilbiie, Florin O and Roland Straub (2013). "Asset market participation, monetary policy rules, and the great inflation". Review of Economics and Statistics 95.2, pp. 377-392.

Bilbiie, Florin Ovidiu and Xavier Ragot (2017). "Optimal monetary policy and liquidity with heterogeneous households".

Blanchard, Olivier and Jordi Galí (2007). "Real Wage Rigidities and the New Keynesian Model". Journal of Money, Credit and Banking 39.s1, pp. 35-65.

Broer, Tobias, Niels-Jakob Harbo Hansen, Per Krusell, and Erik Öberg (2020). "The New Keynesian transmission mechanism: A heterogeneous-agent perspective". The Review of Economic Studies 87.1, pp. 77101.

Campbell, John Y and N Gregory Mankiw (1989). "Consumption, income, and interest rates: Reinterpreting the time series evidence". NBER macroeconomics annual 4, pp. 185-216.

Clarida, Richard, Jordi Gali, and Mark Gertler (1999). "The science of monetary policy: a new Keynesian perspective". Journal of economic literature 37.4, pp. 1661-1707.

Coenen, Günter, Christopher J. Erceg, Charles Freedman, Davide Furceri, Michael Kumhof, René Lalonde, Douglas Laxton, Jesper Lindé, Annabelle Mourougane, Dirk Muir, Susanna Mursula, Carlos de Resende, John Roberts, Werner Roeger, Stephen Snudden, Mathias Trabandt, and Jan in't Veld (2012). "Effects of Fiscal Stimulus in Structural Models". American Economic Journal: Macroeconomics 4.1, pp. $22-68$.

Coibion, Olivier, Yuriy Gorodnichenko, Lorenz Kueng, and John Silvia (2017). "Innocent Bystanders? Monetary Policy and Inequality in the U.S". Journal of Monetary Economics 88.June, pp. 70-89.

Curdia, Vasco and Michael Woodford (2010). "Credit spreads and monetary policy". Journal of Money, credit and Banking 42, pp. 3-35.

De Giorgi, Giacomo and Luca Gambetti (2017). "Business cycle fluctuations and the distribution of consumption". Review of Economic Dynamics 23, pp. 19-41.

Debortoli, Davide and Jordi Gali (2017). "Monetary policy with heterogeneous agents: Insights from TANK models". Manuscript, September.

Dolado, Juan J. B, Gergo Motyovszki, and Evi Pappa (forthcoming). "Monetary Policy and Inequality under Labor Market Frictions and Capital-Skill Complementarity". American Economic Journal: Macroeconomics. 
Eggertsson, Gauti B and Paul Krugman (2012). "Debt, deleveraging, and the liquidity trap: A FisherMinsky-Koo approach". The Quarterly Journal of Economics 127.3, pp. 1469-1513.

Erceg, Christopher J, Dale W Henderson, and Andrew T Levin (2000). "Optimal monetary policy with staggered wage and price contracts". Journal of monetary Economics 46.2, pp. 281-313.

Galí, Jordi (2015). Monetary Policy, Inflation, and the Business Cycle: An Introduction to the New Keynesian Framework and Its Applications. Economics Books 10495. Princeton University Press. URL: https://ideas.repec.org/b/pup/pbooks/10495.html.

Gali, Jordi, J David Lopez-Salido, and Javier Valles (2007). "Understanding the effects of government spending on consumption". Journal of the european economic association 5.1, pp. 227-270.

Gornemann, Nils, Keith Kuester, and Makoto Nakajima (2016). "Doves for the Rich, Hawks for the Poor? Distributional Consequences of Monetary Policy". International Finance Discussion Papers 1167.

Kaplan, Greg, Benjamin Moll, and Giovanni L. Violante (2018). "Monetary Policy According to HANK". American Economic Review 108.3, pp. 697-743.

Nisticò, Salvatore (2016). "Optimal monetary policy and financial stability in a non-Ricardian economy". Journal of the European Economic Association 14.5, pp. 1225-1252.

Ravenna, Federico and Carl E. Walsh (2011). "Welfare-Based Optimal Monetary Policy with Unemployment and Sticky Prices: A Linear-Quadratic Framework". American Economic Journal: Macroeconomics 3.2 , pp. 130-62.

Rotemberg, Julio J and Michael Woodford (1997). "An optimization-based econometric framework for the evaluation of monetary policy". NBER macroeconomics annual 12, pp. 297-346.

Taylor, John B (1993). "Discretion versus policy rules in practice". Carnegie-Rochester conference series on public policy. Vol. 39. Elsevier, pp. 195-214.

Walsh, Carl E (2017). Workers, Capitalists, Wage Flexibility and Welfare. Tech. rep. Mimeo, University of California, Santa Cruz.

Woodford, Michael (2002). "Inflation stabilization and welfare". Contributions in Macroeconomics 2.1.

\section{A Details in Deriving Optimal and RANK-Optimal Policies}

\section{A.1 Deriving the Loss Function}

In this section we approximate the welfare of a utilitarian central planner. There are several steps in this derivation. First, we consider the overall utility within a given period and obtain an expression that involves a linear term in output gap $\hat{Y}_{t}-\hat{A}_{t}$. Second, we take a second order approximation to the New Keynesian Phillips curve that features a linear term in output gap. Finally, we substitute the New Keynesian Phillips curve into the discounted sum of the expression obtained in the first step.

We will often sum all terms independent of monetary policy in the term t.i.p.. We will also use the sign $\approx$ when we take Taylor approximations or ignore terms of order higher than 2 and when we discard t.i.p. terms. Also recall that we define $\hat{x}_{t} \equiv \ln x_{t}-\ln \bar{x}$ and that $\frac{x_{t}-\bar{x}}{\bar{x}} \approx \hat{x}_{t}+\frac{1}{2} \hat{x}_{t}^{2}$.

Step 1: Approximation of Utility Consider the overall utility at time $t$ deriving from consump- 
tion.

$$
\begin{aligned}
\lambda \ln C_{k t}+(1-\lambda) \ln C_{r t} & =\lambda\left(\ln C_{k t}-\ln \bar{C}_{k}\right)+(1-\lambda)\left(\ln C_{r t}-\bar{C}_{r}\right)+\text { t.i.p } \\
& \approx \lambda \hat{C}_{k t}+(1-\lambda) \hat{C}_{r t}
\end{aligned}
$$

Now consider the disutility from labor.

$$
\begin{aligned}
-\chi \frac{N_{t}^{1+\phi}}{1+\phi} & \approx-\chi \bar{N}^{1+\phi}-\chi \bar{N}^{\phi}\left(N_{t}-\bar{N}\right)-\frac{1}{2} \chi \phi \bar{N}^{\phi-1}\left(N_{t}-\bar{N}\right)^{2} \\
& \approx-\chi \bar{N}^{1+\phi}\left[\hat{N}_{i t}+\frac{1+\phi}{2} \hat{N}_{i t}^{2}\right]=-(1-\alpha)\left[\hat{N}_{t}+\frac{1+\phi}{2} \hat{N}_{t}^{2}\right]
\end{aligned}
$$

In the last passage we make use of the steady state efficiency. ${ }^{30}$ At this stage, we can use the production function and rewrite the overall utility as follows.

$$
\begin{aligned}
U_{t}=\lambda \ln C_{k t}+(1-\lambda) \ln C_{r t}-\chi \frac{N_{t}^{1+\phi}}{1+\phi} & \approx \lambda \hat{C}_{k t}+(1-\lambda) \hat{C}_{r t}-(1-\alpha) \hat{N}_{t}-\frac{1+\phi}{2}(1-\alpha) \hat{N}_{t}^{2} \\
& =\lambda \hat{C}_{k t}+(1-\lambda) \hat{C}_{r t}-\left(\hat{Y}_{t}-\hat{A}_{t}\right)-\frac{1}{2} \frac{1+\phi}{1-\alpha}\left(\hat{Y}_{t}-\hat{A}_{t}\right)^{2} \\
& =\lambda \hat{C}_{k t}+(1-\lambda) \hat{C}_{r t}-\hat{Y}_{t}-\frac{1}{2} \frac{1+\phi}{1-\alpha}\left(\hat{Y}_{t}-\hat{A}_{t}\right)^{2}
\end{aligned}
$$

A second order approximation of the aggregate resource constraint equation can be substituted into the above expression.

$$
\begin{gathered}
\hat{Y}_{t}-\frac{\psi}{2} \hat{\Pi}_{t}^{2}=\underbrace{\frac{\lambda \bar{C}_{k}}{\bar{Y}} \hat{C}_{k t}+\underbrace{\frac{(1-\lambda) \bar{C}_{r}}{\bar{Y}}}_{=1-\tilde{\lambda}} \hat{C}_{r t}+\frac{1}{2} \tilde{\lambda}(1-\tilde{\lambda})\left(\hat{C}_{r t}-\hat{C}_{k t}\right)^{2}}_{=\tilde{\lambda}} \\
\tilde{\lambda} \hat{C}_{k t}+(1-\tilde{\lambda}) \hat{C}_{r t}=\hat{Y}_{t}-\frac{\psi_{p}}{2} \hat{\Pi}_{t}^{2}-\frac{1}{2} \tilde{\lambda}(1-\tilde{\lambda})\left(\hat{C}_{r t}-\hat{C}_{k t}\right)^{2}
\end{gathered}
$$

We obtain the following.

$$
\begin{aligned}
U_{t} & \approx-\frac{\psi_{p}}{2} \hat{\Pi}_{t}^{2}-\frac{1}{2} \tilde{\lambda}(1-\tilde{\lambda})\left(\hat{C}_{r t}-\hat{C}_{k t}\right)^{2}-(\lambda-\tilde{\lambda})\left(\hat{C}_{r t}-\hat{C}_{k t}\right)-\frac{1}{2} \frac{1+\phi}{1-\alpha}\left(\hat{Y}_{t}-\hat{A}_{t}\right)^{2} \\
& \approx-\frac{\psi_{p}}{2} \hat{\Pi}_{t}^{2}-\frac{1}{2} \tilde{\lambda}(1-\tilde{\lambda}) \hat{\Delta}_{c t}^{2}-\frac{1}{2} \frac{1+\phi}{1-\alpha}\left(\hat{Y}_{t}-\hat{A}_{t}\right)^{2}-(\lambda-\tilde{\lambda}) \hat{\Delta}_{c t}
\end{aligned}
$$

where we have defined the dynamic inequality term as $\hat{\Delta}_{c t} \equiv \hat{C}_{r t}-\hat{C}_{k t}$. Consider the aggregate resource

\footnotetext{
${ }^{30}$ Specifically, we have used the labor supply condition.
} 
constraint. Define $\tilde{C}_{r t} \equiv \frac{C_{r t}}{Y_{t}}$ and $\tilde{C}_{k t} \equiv \frac{C_{k t}}{Y_{t}}$.

$$
\begin{array}{r}
\tilde{C}_{r t}=-\frac{\lambda \tilde{C}_{k t}}{1-\lambda}+\frac{1}{1-\lambda}-\frac{\psi_{p}\left(\Pi_{t}-1\right)^{2}}{2(1-\lambda)} \\
\hat{\tilde{C}}_{r t}=-\frac{\tilde{\lambda} \hat{\tilde{C}}_{k t}}{1-\tilde{\lambda}}-\frac{1}{2} \frac{\tilde{\lambda} \tilde{\tilde{C}}_{k t}^{2}}{(1-\tilde{\lambda})^{2}}-\frac{1}{2} \frac{\psi_{p}}{1-\tilde{\lambda}} \hat{\Pi}_{t}^{2} \\
\hat{\Delta}_{c t}=\hat{\tilde{C}}_{r t}-\hat{\tilde{C}}_{k t}=-\frac{\hat{\tilde{C}}_{k t}}{1-\tilde{\lambda}}-\frac{1}{2} \frac{\tilde{\lambda} \hat{\tilde{C}}_{k t}^{2}}{(1-\tilde{\lambda})^{2}}-\frac{1}{2} \frac{\psi_{p}}{1-\tilde{\lambda}} \hat{\Pi}_{t}^{2}
\end{array}
$$

At this stage, the have the following.

$$
U_{t}=-\frac{1}{2} \psi_{p} \hat{\Pi}_{t}^{2}-\frac{1}{2} \tilde{\lambda}(1-\tilde{\lambda}) \hat{\Delta}_{c t}^{2}-\frac{1}{2} \frac{1+\phi}{1-\alpha}\left(\hat{Y}_{t}-\hat{A}_{t}\right)^{2}+\frac{(\lambda-\tilde{\lambda})}{1-\tilde{\lambda}}\left[\hat{\tilde{C}}_{k t}+\frac{1}{2} \frac{\tilde{\lambda}}{1-\tilde{\lambda}} \hat{\tilde{C}}_{k t}^{2}+\frac{1}{2} \psi_{p} \hat{\Pi}_{t}^{2}\right]
$$

Now, the goal is to substitute away terms in $\hat{\tilde{C}}_{k t}$. Define the labor share variable $L S_{t}=\frac{w_{t} N_{t}}{Y_{t}}$ (whose steady state value is $1-\alpha$ ) and consider the budget constraint of the Keynesian agent. ${ }^{31}$ We take logarithms on both sides, subtract $\ln \overline{\tilde{C}}_{k}$, and take a second order approximation.

$$
\begin{aligned}
\tilde{C}_{k t} & =(1-\tau) \delta\left(1+T_{p}\right)-T_{p}+\left[\left(\frac{A_{t}}{\bar{A}}\right)^{-\gamma}-(1-\tau) \delta\right] L S_{t}-\frac{\psi_{p}}{2}(1-\tau) \delta\left(\Pi_{t}-1\right)^{2} \\
\hat{\tilde{C}}_{k t} & =\ln \left\{\left(1-\tau_{t r}\right) \delta\left(1+T_{p}\right)-T_{p}+\left[\left(\frac{A_{t}}{\bar{A}}\right)^{-\gamma}-\left(1-\tau_{t r}\right) \delta\right] L S_{t}-\frac{\psi_{p}}{2}\left(1-\tau_{t r}\right) \delta\left(\Pi_{t}-\bar{\Pi}\right)^{2}+\left(\tau_{t r}-\tau\right) \frac{\delta \bar{d}}{Y_{t}}\right\}- \\
& -\ln \overline{\tilde{C}}_{k}
\end{aligned}
$$

A second order approximation gives the following.

$$
\begin{aligned}
\hat{\tilde{C}}_{k t} & =-\frac{\gamma(1-\alpha)}{\tilde{\tilde{C}}_{k}} \hat{A}_{t}+\frac{(1-\alpha)[1-(1-\tau) \delta]}{\tilde{\tilde{C}}_{k}} \hat{S}_{t}+ \\
& +\left\{\frac{1}{2} \gamma^{2} \frac{1-\alpha}{\tilde{\tilde{C}}_{k}}\left(1-\frac{1-\alpha}{\tilde{\tilde{C}}_{k}}\right)\right\} \hat{A}_{t}^{2}+\left\{\frac{1}{2} \frac{[1-(1-\tau) \delta](1-\alpha)}{\tilde{\tilde{C}}_{k}}\left[1-\frac{[1-(1-\tau) \delta](1-\alpha)}{\tilde{C}_{k}}\right]\right\} \hat{L} \hat{S}_{t}^{2}+ \\
& -\frac{1}{2} \frac{(1-\tau) \delta \psi_{p}}{\tilde{\tilde{C}}_{k}} \hat{\Pi}_{t}^{2}-\gamma \frac{1-\alpha}{\tilde{C}_{k}}\left[1-\frac{[1-(1-\tau) \delta](1-\alpha)}{\tilde{\tilde{C}}_{k}}\right] \hat{A}_{t} \hat{L S} S_{t}
\end{aligned}
$$

Notice that for our purpose, we can disregard the t.i.p. terms and keep the terms up to second order. We

\footnotetext{
${ }^{31}$ To obtain the expression that follows, we have used the labor supply equation (6), the dividends equation (9), and the transfer equation (12).
} 
then can use the following in the welfare function.

$$
\begin{aligned}
& \hat{\tilde{C}}_{k t}=\frac{(1-\alpha)[1-(1-\tau) \delta]}{\tilde{C}_{k}} \hat{S}_{t}+\left\{\frac{1}{2} \frac{[1-(1-\tau) \delta](1-\alpha)}{\tilde{\tilde{C}}_{k}}\left[1-\frac{[1-(1-\tau) \delta](1-\alpha)}{\tilde{\tilde{C}}_{k}}\right]\right\} \hat{L S_{t}^{2}}+ \\
& -\frac{1}{2} \frac{(1-\tau) \delta \psi_{p}}{\tilde{\tilde{C}}_{k}} \hat{\Pi}_{t}^{2}-\gamma \frac{1-\alpha}{\tilde{\tilde{C}}_{k}}\left[1-\frac{[1-(1-\tau) \delta](1-\alpha)}{\tilde{\tilde{C}}_{k}}\right] \hat{A}_{t} \hat{L S_{t}} \\
& \hat{\tilde{C}}_{k t}^{2}=\left[\frac{(1-\alpha)[1-(1-\tau) \delta]}{\tilde{\tilde{C}}_{k}}\right]^{2} \hat{L S_{t}^{2}}-2 \gamma \frac{1-\alpha}{\tilde{\tilde{C}}_{k}} \frac{[1-(1-\tau) \delta](1-\alpha)}{\tilde{\tilde{C}}_{k}} \hat{A}_{t} \hat{L S} S_{t}
\end{aligned}
$$

Notice that under the baseline assumptions, we have that $\hat{L S} S_{t}=\frac{1+\phi}{1-\alpha}\left(\hat{Y}_{t}-\hat{A}_{t}\right)$. At this stage, the welfare function is the following.

$$
\begin{aligned}
U_{t} & =-\frac{1}{2} \psi_{p} \hat{\Pi}_{t}^{2}-\frac{1}{2} \tilde{\lambda}(1-\tilde{\lambda}) \hat{\Delta}_{c t}^{2}-\frac{1}{2} \frac{1+\phi}{1-\alpha}\left(\hat{Y}_{t}-\hat{A}_{t}\right)^{2}+\frac{(\lambda-\tilde{\lambda})}{1-\tilde{\lambda}}\left[\frac{(1-\alpha)[1-(1-\tau) \delta]}{\tilde{C}_{k}} \frac{1+\phi}{1-\alpha}\left(\hat{Y}_{t}-\hat{A}_{t}\right)\right]+ \\
& +\frac{(\lambda-\tilde{\lambda})}{1-\tilde{\lambda}}\left[\frac{1}{2} \frac{[1-(1-\tau) \delta](1-\alpha)}{\tilde{C}_{k}}\left[1-\frac{[1-(1-\tau) \delta](1-\alpha)}{\tilde{C}_{k}}\right]\left(\frac{1+\phi}{1-\alpha}\right)^{2}\left(\hat{Y}_{t}-\hat{A}_{t}\right)^{2}\right]+ \\
& +\frac{(\lambda-\tilde{\lambda})}{1-\tilde{\lambda}}\left[-\frac{1}{2} \frac{(1-\tau) \delta \psi_{p}}{\tilde{C}_{k}} \hat{\Pi}_{t}^{2}-\gamma \frac{1-\alpha}{\tilde{C}_{k}}\left[1-\frac{[1-(1-\tau) \delta](1-\alpha)}{\tilde{C}_{k}}\right] \hat{A}_{t} \frac{1+\phi}{1-\alpha}\left(\hat{Y}_{t}-\hat{A}_{t}\right)\right]+ \\
& +\frac{(\lambda-\tilde{\lambda})}{1-\tilde{\lambda}} \frac{1}{2} \frac{\tilde{\lambda}}{1-\tilde{\lambda}}\left[\left[\frac{(1-\alpha)[1-(1-\tau) \delta]}{\tilde{C}_{k}}\right]^{2}\left(\frac{1+\phi}{1-\alpha}\right)^{2}\left(\hat{Y}_{t}-\hat{A}_{t}\right)^{2}-2 \gamma \frac{1-\alpha}{\tilde{C}_{k}} \frac{[1-(1-\tau) \delta](1-\alpha)}{\tilde{C}_{k}} \hat{A}_{t} \frac{1+\phi}{1-\alpha}\left(\hat{Y}_{t}-\hat{A}_{t}\right)\right] \\
& +\frac{(\lambda-\tilde{\lambda})}{1-\tilde{\lambda}}\left[\frac{1}{2} \psi_{p} \hat{\Pi}_{t}^{2}\right]
\end{aligned}
$$


We can collect terms and have the following coefficients.

$$
\begin{aligned}
& \hat{\Pi}_{t}^{2}:-\frac{1}{2} \psi_{p}\left[1-\frac{(\lambda-\tilde{\lambda})}{1-\tilde{\lambda}}\left[1-\frac{(1-\tau) \delta}{\tilde{C}_{k}}\right]\right] \\
& \hat{\Delta}_{c t}^{2}:-\frac{1}{2} \tilde{\lambda}(1-\tilde{\lambda}) \\
& \hat{Y}_{t}^{2}:-\frac{1}{2} \frac{1+\phi}{1-\alpha}+\frac{1}{2} \frac{(\lambda-\tilde{\lambda})}{1-\tilde{\lambda}} \frac{[1-(1-\tau) \delta](1-\alpha)}{\tilde{\tilde{C}}_{k}}\left[1-\frac{1-2 \tilde{\lambda}}{1-\tilde{\lambda}} \frac{[1-(1-\tau) \delta](1-\alpha)}{\tilde{\tilde{C}}_{k}}\right]\left(\frac{1+\phi}{1-\alpha}\right)^{2} \equiv-\frac{1}{2} \tilde{W}_{Y Y} \\
& \hat{A}_{t} \hat{Y}_{t}: \frac{1+\phi}{1-\alpha}-\frac{(\lambda-\tilde{\lambda})}{1-\tilde{\lambda}} \frac{[1-(1-\tau) \delta](1-\alpha)}{\tilde{\tilde{C}}_{k}}\left[1-\frac{1-2 \tilde{\lambda}}{1-\tilde{\lambda}} \frac{[1-(1-\tau) \delta](1-\alpha)}{\tilde{\tilde{C}}_{k}}\right]\left(\frac{1+\phi}{1-\alpha}\right)^{2} \\
& -\frac{(\lambda-\tilde{\lambda})}{1-\tilde{\lambda}} \gamma \frac{1-\alpha}{\tilde{C}_{k}}\left[1-\frac{1-2 \tilde{\lambda}}{1-\tilde{\lambda}} \frac{[1-(1-\tau) \delta](1-\alpha)}{\tilde{C}_{k}}\right] \frac{1+\phi}{1-\alpha} \\
& \equiv-\frac{1}{2} W_{\Pi} \\
& \equiv-\frac{1}{2} W_{\Delta_{c}} \\
& \hat{Y}_{t}: \frac{(\lambda-\tilde{\lambda})}{1-\tilde{\lambda}} \frac{[1-(1-\tau) \delta](1-\alpha)}{\tilde{\tilde{C}}_{k}} \frac{1+\phi}{1-\alpha} \\
& \mathbf{W}=\sum_{t=0}^{\infty} \beta^{t}\left\{-\frac{1}{2} \tilde{W}_{\Pi} \hat{\Pi}_{t}^{2}-\frac{1}{2} W_{\Delta_{c}} \hat{\Delta}_{t}^{2}-\frac{1}{2} \tilde{W}_{Y Y} \hat{Y}_{t}^{2}+\tilde{W}_{A Y} \hat{A}_{t} \hat{Y}_{t}+\tilde{W}_{Y} \hat{Y}_{t}\right\} \\
& \equiv \tilde{W}_{A Y} \\
& \equiv \tilde{W}_{Y}
\end{aligned}
$$

Step 2: Approximation of New Keynesian Phillips curve The second order approximation to the New Keynesian Phillips curve is derived. ${ }^{32}$

$$
Y_{t} C_{r t}^{-1}\left(\frac{\Pi_{t}^{2}-\Pi_{t}}{\overline{Y C_{r}^{-1}}}\right)=\beta C_{r t+1}^{-1} Y_{t+1} \frac{\Pi_{t}^{2}-\Pi_{t}}{\overline{Y C_{r}^{-1}}}+\frac{\theta_{p}}{\psi_{p} \overline{Y C_{r}^{-1}}}\left(\frac{1}{1-\alpha} L S_{t}-1\right) Y_{t} C_{r t}^{-1}
$$

We break the derivation in 3 parts. On the LHS we have the following.

$$
\tilde{T}_{t}=\hat{\Pi}_{t}+\frac{3}{2} \hat{\Pi}_{t}^{2}+\hat{\Pi}_{t} \hat{Y}_{t}-\hat{\Pi}_{t} \hat{C}_{r t}
$$

For the first part of the RHS we similarly have the following.

$$
\tilde{T}_{t+1}=\hat{\Pi}_{t+1}+\frac{3}{2} \hat{\Pi}_{t+1}^{2}+\hat{\Pi}_{t+1} \hat{Y}_{t+1}-\hat{\Pi}_{t+1} \hat{C}_{r t+1}
$$

For the second part on the RHS we have the following.

$$
T T_{t}=\frac{\theta_{p}}{\psi_{p}}\left\{\hat{L S_{t}}+\frac{1}{2} \hat{L S_{t}^{2}}+\hat{L S_{t}} \hat{Y}_{t}-\hat{L S_{t}} \hat{C}_{r t}\right\}
$$

Now we can make the use of some previous results, namely that, to a first order, $\hat{C}_{r t}=\hat{\Delta}_{c t}+\hat{C}_{k t}=-\frac{\hat{\tilde{C}}_{k t}}{1-\tilde{\lambda}}+$

\footnotetext{
${ }^{32}$ For notational convenience, expectation operators are dropped.
} 
$\hat{\tilde{C}}_{k t}+\hat{Y}_{t}=-\frac{\tilde{\lambda}}{1-\tilde{\lambda}} \hat{\tilde{C}}_{k t}+\hat{Y}_{t}$. The term in the Phillips curve then becomes the following.

$$
T T_{t}=\frac{\theta_{p}}{\psi_{p}}\left\{\hat{L} S_{t}+\frac{1}{2} \hat{L S}_{t}^{2}+\frac{\tilde{\lambda}}{1-\tilde{\lambda}} \hat{\tilde{C}}_{k t} \hat{L S} S_{t}\right\}
$$

Finally, taking a first order approximation on $\hat{\tilde{C}}_{k t}$, the above becomes:

$$
T T_{t}=\frac{\theta_{p}}{\psi_{p}}\left\{\hat{L} S_{t}+\left(\frac{1}{2}+\frac{\tilde{\lambda}}{1-\tilde{\lambda}} \frac{(1-\alpha)(1-(1-\tau) \delta)}{\tilde{\tilde{C}}_{k}}\right) \hat{L S}_{t}^{2}+\frac{\tilde{\lambda}}{1-\tilde{\lambda}} \hat{L S_{t}}\left[-\gamma \frac{(1-\alpha)}{\tilde{\tilde{C}}_{k}} \hat{A}_{t}\right]\right\}
$$

Collecting all the three parts we obtain the following.

$$
\begin{aligned}
\frac{\psi_{p}}{\theta_{p}} \tilde{T}_{0} & =\sum_{t=0}^{\infty} \beta^{t}\left\{\hat{L} S_{t}+\left(\frac{1}{2}+\frac{\tilde{\lambda}}{1-\tilde{\lambda}} \frac{(1-\alpha)(1-(1-\tau) \delta)}{\tilde{C}_{k}}\right) \hat{L} S_{t}^{2}-\frac{\tilde{\lambda}}{1-\tilde{\lambda}} \gamma \frac{(1-\alpha)}{\tilde{C}_{k}} \hat{A}_{t} \hat{L S_{t}}\right\} \\
\sum_{t=0}^{\infty} \beta^{t} \hat{L S} S_{t} & =\frac{\psi_{p}}{\theta_{p}} \tilde{T}_{0}+\sum_{t=0}^{\infty} \beta^{t}\left\{-\left(\frac{1}{2}+\frac{\tilde{\lambda}}{1-\tilde{\lambda}} \frac{(1-\alpha)(1-(1-\tau) \delta)}{\tilde{\tilde{C}}_{k}}\right) \hat{L S_{t}^{2}}+\frac{\tilde{\lambda}}{1-\tilde{\lambda}} \gamma \frac{(1-\alpha)}{\tilde{\tilde{C}}_{k}} \hat{A}_{t} \hat{L S}\right\}
\end{aligned}
$$

The next step is multiply the above expression by $\frac{(\lambda-\tilde{\lambda})}{1-\tilde{\lambda}} \frac{[1-(1-\tau) \delta](1-\alpha)}{\tilde{\tilde{C}}_{k}}$. This transforms the Phillips curve in a very convenient form. In this passage we have used that the labor share is proportional to the output gap.

$$
\begin{aligned}
\sum_{t=0}^{\infty} \beta^{t} \tilde{W}_{Y}\left(\hat{Y}_{t}-\hat{A}_{t}\right) & =\frac{\psi_{p}}{\theta_{p}} \frac{(\lambda-\tilde{\lambda})}{1-\tilde{\lambda}} \frac{[1-(1-\tau) \delta](1-\alpha)}{\tilde{C}_{k}} \tilde{T}_{0}+ \\
& +\frac{(\lambda-\tilde{\lambda})}{1-\tilde{\lambda}} \frac{[1-(1-\tau) \delta](1-\alpha)}{\tilde{C}_{k}} \sum_{t=0}^{\infty} \beta^{t}\left\{-\left(\frac{1}{2}+\frac{\tilde{\lambda}}{1-\tilde{\lambda}} \frac{(1-\alpha)(1-(1-\tau) \delta)}{\tilde{C}_{k}}\right)\left(\frac{1+\phi}{1-\alpha}\right)^{2}\left(\hat{Y}_{t}-\hat{A}_{t}\right)^{2}\right\} \\
& +\frac{(\lambda-\tilde{\lambda})}{1-\tilde{\lambda}} \frac{[1-(1-\tau) \delta](1-\alpha)}{\tilde{C}_{k}} \sum_{t=0}^{\infty} \beta^{t}\left\{\frac{\tilde{\lambda}}{1-\tilde{\lambda}} \gamma \frac{(1-\alpha)}{\tilde{C}_{k}} \frac{1+\phi}{1-\alpha} \hat{A}_{t}\left(\hat{Y}_{t}-\hat{A}_{t}\right)\right\}
\end{aligned}
$$

We define $T_{0} \equiv \frac{\psi_{p}}{\theta_{p}} \frac{(\lambda-\tilde{\lambda})}{1-\tilde{\lambda}} \frac{[1-(1-\tau) \delta](1-\alpha)}{\tilde{C}_{k}} \tilde{T}_{0}$, and can rewrite the above expression ignoring t.i.p. terms.

$$
\begin{aligned}
\sum_{t=0}^{\infty} \beta^{t} \tilde{W}_{Y} \hat{Y}_{t} & =T_{0}+\text { t.i.p.+ } \\
& +\frac{(\lambda-\tilde{\lambda})}{1-\tilde{\lambda}} \frac{[1-(1-\tau) \delta](1-\alpha)}{\tilde{C}_{k}} \sum_{t=0}^{\infty} \beta^{t}\left\{-\left(\frac{1}{2}+\frac{\tilde{\lambda}}{1-\tilde{\lambda}} \frac{(1-\alpha)(1-(1-\tau) \delta)}{\tilde{C}_{k}}\right)\left(\frac{1+\phi}{1-\alpha}\right)^{2}\left(\hat{Y}_{t}-\hat{A}_{t}\right)^{2}\right\} \\
& +\frac{(\lambda-\tilde{\lambda})}{1-\tilde{\lambda}} \frac{[1-(1-\tau) \delta](1-\alpha)}{\tilde{\tilde{C}}_{k}} \sum_{t=0}^{\infty} \beta^{t}\left\{\frac{\tilde{\lambda}}{1-\tilde{\lambda}} \gamma \frac{(1-\alpha)}{\tilde{C}_{k}} \frac{1+\phi}{1-\alpha} \hat{A}_{t} \hat{Y}_{t}\right\}
\end{aligned}
$$

Step 3: Substitution of New Keynesian Phillips curve in Welfare function The final step 
involves substituting the New Keynesian Phillips curve into the welfare function.

$$
\begin{aligned}
\mathbf{W} & =\sum_{t=0}^{\infty} \beta^{t}\left\{-\frac{1}{2} W_{\Pi} \hat{\Pi}_{t}^{2}-\frac{1}{2} W_{\Delta_{c}} \hat{\Delta}_{t}^{2}-\frac{1}{2} \tilde{W}_{Y Y} \hat{Y}_{t}^{2}+\tilde{W}_{A Y} \hat{A}_{t} \hat{Y}_{t}+\tilde{W}_{Y} \hat{Y}_{t}\right\} \\
& =\sum_{t=0}^{\infty} \beta^{t}\left\{-\frac{1}{2} W_{\Pi} \hat{\Pi}_{t}^{2}-\frac{1}{2} W_{\Delta_{c}} \hat{\Delta}_{t}^{2}-\frac{1}{2} \tilde{W}_{Y^{2}} \hat{Y}_{t}^{2}+\tilde{W}_{A Y} \hat{A}_{t} \hat{Y}_{t}\right\}+T_{0}+ \\
& +\sum_{t=0}^{\infty} \beta^{t}\left\{\frac{(\lambda-\tilde{\lambda})}{1-\tilde{\lambda}} \frac{[1-(1-\tau) \delta](1-\alpha)}{\tilde{C}_{k}}\left\{-\left(\frac{1}{2}+\frac{\tilde{\lambda}}{1-\tilde{\lambda}} \frac{(1-\alpha)(1-(1-\tau) \delta)}{\tilde{C}_{k}}\right)\left(\frac{1+\phi}{1-\alpha}\right)^{2}\left(\hat{Y}_{t}-\hat{A}_{t}\right)^{2}\right\}\right\}+ \\
& +\sum_{t=0}^{\infty} \beta^{t}\left\{\frac{(\lambda-\tilde{\lambda})}{1-\tilde{\lambda}} \frac{[1-(1-\tau) \delta](1-\alpha)}{\tilde{C}_{k}} \frac{\tilde{\lambda}}{1-\tilde{\lambda}} \gamma \frac{(1-\alpha)}{\tilde{C}_{k}} \frac{1+\phi}{1-\alpha} \hat{A}_{t} \hat{Y}_{t}\right\} \\
\mathbf{W} & =\sum_{t=0}^{\infty} \beta^{t}\left\{-\frac{1}{2} W_{\Pi} \hat{\Pi}_{t}^{2}-\frac{1}{2} W_{\Delta_{c}} \hat{\Delta}_{t}^{2}-\frac{1}{2} W_{Y} \hat{Y}_{t}^{2}+W_{A Y} \hat{A}_{t} \hat{Y}_{t}\right\}+T_{0}
\end{aligned}
$$

where

$$
\begin{aligned}
-\frac{1}{2} W_{Y} & =-\frac{1}{2} \tilde{W}_{Y Y}-\frac{1}{2}\left(1+2 \frac{\tilde{\lambda}}{1-\tilde{\lambda}} \frac{(1-\alpha)(1-(1-\tau) \delta)}{\tilde{\tilde{C}}_{k}}\right)\left(\frac{1+\phi}{1-\alpha}\right)^{2} \frac{(\lambda-\tilde{\lambda})}{1-\tilde{\lambda}} \frac{[1-(1-\tau) \delta](1-\alpha)}{\tilde{\tilde{C}}_{k}}= \\
& =-\frac{1}{2} \frac{1+\phi}{1-\alpha}-\frac{1}{2} \frac{(\lambda-\tilde{\lambda})}{(1-\tilde{\lambda})^{2}}\left(\frac{(1-\alpha)(1-(1-\tau) \delta)}{\tilde{C}_{k}}\right)^{2}\left(\frac{1+\phi}{1-\alpha}\right)^{2} \\
W_{A Y} & =\tilde{W}_{A Y}+\frac{(\lambda-\tilde{\lambda})}{1-\tilde{\lambda}} \frac{[1-(1-\tau) \delta](1-\alpha)}{\tilde{\tilde{C}}_{k}} \frac{\tilde{\lambda}}{1-\tilde{\lambda}} \gamma \frac{(1-\alpha)}{\tilde{\tilde{C}}_{k}} \frac{1+\phi}{1-\alpha}+ \\
& +\left(1+2 \frac{\tilde{\lambda}}{1-\tilde{\lambda}} \frac{(1-\alpha)(1-(1-\tau) \delta)}{\tilde{\tilde{C}}_{k}}\right)\left(\frac{1+\phi}{1-\alpha}\right)^{2} \frac{(\lambda-\tilde{\lambda})}{1-\tilde{\lambda}} \frac{[1-(1-\tau) \delta](1-\alpha)}{\tilde{\tilde{C}}_{k}}= \\
& =\frac{1+\phi}{1-\alpha}+\frac{(\lambda-\tilde{\lambda})}{(1-\tilde{\lambda})^{2}}\left[\frac{[1-(1-\tau) \delta](1-\alpha)}{\tilde{\tilde{C}}_{k}}\right]^{2}\left(\frac{1+\phi}{1-\alpha}\right)^{2}-\frac{(\lambda-\tilde{\lambda})}{1-\tilde{\lambda}} \gamma \frac{1-\alpha}{\tilde{C}_{k}}\left[1-\frac{[1-(1-\tau) \delta](1-\alpha)}{\tilde{C}_{k}}\right] \frac{1+\phi}{1-\alpha}
\end{aligned}
$$

Finally, we can write the welfare function as follows.

$$
\begin{aligned}
& \mathbf{W}=\sum_{t=0}^{\infty} \beta^{t}\left\{-\frac{1}{2} W_{\Pi} \hat{\Pi}_{t}^{2}-\frac{1}{2} W_{\Delta_{c}} \hat{\Delta}_{c t}^{2}-\frac{1}{2} W_{Y}\left(\hat{Y}_{t}-\hat{Y}_{t}^{*}\right)^{2}\right\}+T_{0} \\
& \hat{Y}_{t}^{*} \equiv \frac{W_{A Y}}{W_{Y}} \hat{A}_{t}
\end{aligned}
$$

Notice that in the welfare function we have output deviations from $\hat{Y}_{t}^{*}$, not the natural level itself, i.e. the central bank has a different "output target". Below, we report the final welfare weights that are derived in 
the previous passages.

$$
\begin{aligned}
W_{\Pi} & =\psi_{p}\left[1-\frac{(\lambda-\tilde{\lambda})}{1-\tilde{\lambda}}\left[1-\frac{(1-\tau) \delta}{\tilde{C}_{k}}\right]\right] \\
W_{\Delta_{c}} & =\tilde{\lambda}(1-\tilde{\lambda}) \\
W_{Y} & =\frac{1+\phi}{1-\alpha}+\frac{(\lambda-\tilde{\lambda})}{(1-\tilde{\lambda})^{2}}\left(\frac{(1-\alpha)(1-(1-\tau) \delta)}{\tilde{C}_{k}}\right)^{2}\left(\frac{1+\phi}{1-\alpha}\right)^{2}
\end{aligned}
$$

where $\tilde{\lambda} \equiv \lambda \frac{\bar{C}_{k}}{\bar{Y}}=\lambda \bar{C}_{k}$. From the budget constraint of Keynesian agents $(4), \bar{C}_{k}=\bar{w} \bar{N}-T_{p} \bar{Y}+(1-\tau) \delta \bar{d}$ where the steady state levels of wage, labor, output and dividends do not depend on the parameters that govern inequality, $\tau$ or $\gamma$.

\section{A.2 The Central Bank's Maximization Problem}

The problem is the following.

$$
\max \mathbf{W}=\sum_{t=0}^{\infty} \beta^{t}\left\{-\frac{1}{2} W_{\Pi} \hat{\Pi}_{t}^{2}-\frac{1}{2} W_{\Delta_{c}} \hat{\Delta}_{c t}^{2}-\frac{1}{2} W_{Y}\left(\hat{Y}_{t}-\hat{Y}_{t}^{*}\right)^{2}\right\}+T_{0}
$$

subject to the New-Keynesian Phillips curve, and the dynamic inequality equation (both to a first order approximation).

$$
\begin{aligned}
\hat{\Pi}_{t} & =\beta E_{t} \hat{\Pi}_{t+1}+\frac{\theta_{p}}{\psi} \frac{1+\phi}{1-\alpha}\left(\hat{Y}_{t}-\hat{Y}_{t}^{*}\right)+\text { t.i.p. } \\
\hat{C}_{r t}-\hat{C}_{k t} & =-\frac{1}{1-\tilde{\lambda}} \frac{1-(1-\tau) \delta}{\tilde{C}_{k}}(1-\alpha) \frac{1+\phi}{1-\alpha}\left(\hat{Y}_{t}-\hat{Y}_{t}^{*}\right)+\text { t.i.p. }
\end{aligned}
$$

The solution can be obtained as the solution to a Lagrangian, with an extra constraint to the value of $T_{0}$ (what the timeless commitment basically sets).

$$
\begin{aligned}
\mathbf{L} & =\sum_{t=0}^{\infty} \beta^{t}\left\{-\frac{1}{2} W_{\Pi} \hat{\Pi}_{t}^{2}-\frac{1}{2} W_{\Delta_{c}} \hat{\Delta}_{t}^{2}-\frac{1}{2} W_{Y}\left(\hat{Y}_{t}-\hat{Y}_{t}^{*}\right)^{2}\right\}+ \\
& +\sum_{t=0}^{\infty} \beta^{t} \mu_{t}\left[\hat{\Pi}_{t}-\beta E_{t} \hat{\Pi}_{t+1}-\frac{\theta_{p}}{\psi} \frac{1+\phi}{1-\alpha}\left(\hat{Y}_{t}-\hat{Y}_{t}^{*}\right)\right]+ \\
& +\sum_{t=0}^{\infty} \beta^{t} \eta_{t}\left[\hat{\Delta}_{t}+\frac{1}{1-\tilde{\lambda}} \frac{1-(1-\tau) \delta}{\tilde{C}_{k}}(1-\alpha) \frac{1+\phi}{1-\alpha}\left(\hat{Y}_{t}-\hat{Y}_{t}^{*}\right)\right]
\end{aligned}
$$

FOCs follow.

$$
\begin{array}{r}
-W_{\Pi} \hat{\Pi}_{t}+\mu_{t}-\mu_{t-1}=0 \\
-W_{\Delta_{c}} \hat{\Delta}_{t}+\eta_{t}=0 \\
-W_{Y}\left(\hat{Y}_{t}-\hat{Y}_{t}^{*}\right)-\mu_{t} \frac{\theta_{p}}{\psi} \frac{1+\phi}{1-\alpha}+\eta_{t} \frac{1}{1-\tilde{\lambda}} \frac{1-(1-\tau) \delta}{\tilde{C}_{k}}(1-\alpha) \frac{1+\phi}{1-\alpha}
\end{array}
$$


The FOCs boil down to a single equation.

$$
\begin{array}{r}
-W_{\Pi} \hat{\Pi}_{t}-\frac{W_{Y}}{\frac{\theta_{p}}{\psi} \frac{1+\phi}{1-\alpha}}\left(\hat{Y}_{t}-\hat{Y}_{t}^{*}\right)+\frac{\frac{1}{1-\tilde{\lambda}} \frac{1-(1-\tau) \delta}{\tilde{C}_{k}}(1-\alpha) \frac{1+\phi}{1-\alpha} W_{\Delta_{c}}}{\frac{\theta_{p}}{\psi} \frac{1+\phi}{1-\alpha}} \hat{\Delta}_{t}+ \\
+\frac{W_{Y}}{\frac{\theta_{p}}{\psi} \frac{1+\phi}{1-\alpha}}\left(\hat{Y}_{t-1}-\hat{Y}_{t-1}^{*}\right)-\frac{\frac{1}{1-\hat{\lambda}} \frac{1-(1-\tau) \delta}{\tilde{\bar{C}}_{k}}(1-\alpha) \frac{1+\phi}{1-\alpha} W_{\Delta_{c}}}{\frac{\theta_{p}}{\psi} \frac{1+\phi}{1-\alpha}} \hat{\Delta}_{t-1}=0
\end{array}
$$

\section{A.3 Details in Deriving RANK-Optimal Monetary Policy}

In this Section, we explain the problem of a RANK central bank.

If all agents are Ricardian, meaning $\lambda=\tilde{\lambda}=0$, the weights on inflation and output gap defined in ?? simplify to:

$$
\begin{aligned}
& W_{\Pi}=\psi_{p}\left[1-\frac{(\lambda-\tilde{\lambda})}{1-\tilde{\lambda}}\left[1-\frac{(1-\tau) \delta}{\tilde{C}_{k}}\right]\right] \\
& \tilde{W}_{Y Y}=\frac{1+\phi}{1-\alpha}+\frac{1}{2} \frac{(\lambda-\tilde{\lambda})}{1-\tilde{\lambda}} \frac{[1-(1-\tau) \delta](1-\alpha)}{\tilde{C}_{k}}\left[1-\frac{1-2 \tilde{\lambda}}{1-\tilde{\lambda}} \frac{[1-(1-\tau) \delta](1-\alpha)}{\tilde{C}_{k}}\right]\left(\frac{1+\phi}{1-\alpha}\right)^{2}=\frac{1+\phi}{1-\alpha} \\
& \tilde{W}_{A Y}=\frac{1+\phi}{1-\alpha}-\frac{(\lambda-\tilde{\lambda})}{1-\tilde{\lambda}} \frac{[1-(1-\tau) \delta](1-\alpha)}{\tilde{\tilde{C}}_{k}}\left[1-\frac{1-2 \tilde{\lambda}}{1-\tilde{\lambda}} \frac{[1-(1-\tau) \delta](1-\alpha)}{\tilde{C}_{k}}\right]\left(\frac{1+\phi}{1-\alpha}\right)^{2} \\
& -\frac{(\lambda-\tilde{\lambda})}{1-\tilde{\lambda}} \gamma \frac{1-\alpha}{\tilde{C}_{k}}\left[1-\frac{1-2 \tilde{\lambda}}{1-\tilde{\lambda}} \frac{[1-(1-\tau) \delta](1-\alpha)}{\tilde{C}_{k}}\right] \frac{1+\phi}{1-\alpha} \quad=\frac{1+\phi}{1-\alpha} \\
& \begin{aligned}
\tilde{W}_{Y} & =\frac{(\lambda-\tilde{\lambda})}{1-\tilde{\lambda}} \frac{[1-(1-\tau) \delta](1-\alpha)}{\tilde{C}_{k}} \frac{1+\phi}{1-\alpha} \\
W_{A Y} & =\tilde{W}_{A Y}+\frac{(\lambda-\tilde{\lambda})}{1-\tilde{\lambda}} \frac{[1-(1-\tau) \delta](1-\alpha)}{\tilde{\tilde{C}}_{k}} \frac{\tilde{\lambda}}{1-\tilde{\lambda}} \gamma \frac{(1-\alpha)}{\tilde{\tilde{C}}_{k}} \frac{1+\phi}{1-\alpha}+
\end{aligned} \\
& +\left(1+2 \frac{\tilde{\lambda}}{1-\tilde{\lambda}} \frac{(1-\alpha)(1-(1-\tau) \delta)}{\tilde{C}_{k}}\right)\left(\frac{1+\phi}{1-\alpha}\right)^{2} \frac{(\lambda-\tilde{\lambda})}{1-\tilde{\lambda}} \frac{[1-(1-\tau) \delta](1-\alpha)}{\tilde{C}_{k}} \quad=\frac{1+\phi}{1-\alpha} \\
& W_{Y}=\frac{1+\phi}{1-\alpha}+\frac{(\lambda-\tilde{\lambda})}{(1-\tilde{\lambda})^{2}}\left(\frac{(1-\alpha)(1-(1-\tau) \delta)}{\tilde{C}_{k}}\right)^{2}\left(\frac{1+\phi}{1-\alpha}\right)^{2} \quad=\frac{1+\phi}{1-\alpha}
\end{aligned}
$$

The welfare function can then be written as follows.

$$
\mathbf{W}=\sum_{t=0}^{\infty} \beta^{t}\left\{-\frac{1}{2} \psi_{p} \hat{\Pi}_{t}^{2}-\frac{1}{2} \frac{1+\phi}{1-\alpha}\left(\hat{Y}_{t}-\hat{A}_{t}\right)^{2}\right\}
$$

subject to the Phillips curve

$$
\hat{\Pi}_{t}=\beta E_{t} \hat{\Pi}_{t+1}+\frac{\theta_{p}}{\psi_{p}} \frac{1+\phi}{1-\alpha}\left(\hat{Y}_{t}-\hat{A}_{t}\right)
$$


And the restriction that $T_{0}$ equals the level attained under OMP, i.e. when solving (34).

The first order conditions follow.

$$
\begin{array}{r}
-\beta^{t} \psi_{p} \hat{\Pi}_{t}+\beta^{t} \mu_{t}-\beta^{t-1} \beta \mu_{t-1}=0 \\
-\beta^{t} \frac{1+\phi}{1-\alpha}\left(\hat{Y}_{t}-\hat{A}_{t}\right)-\beta^{t} \mu_{t} \frac{\theta_{p}}{\psi_{p}} \frac{1+\phi}{1-\alpha}=0
\end{array}
$$

Rearranging we obtain the following.

$$
\begin{aligned}
-\psi_{p} \hat{\Pi}_{t}+\mu_{t}-\mu_{t-1} & =0 \\
-\frac{1}{\frac{\theta_{p}}{\psi_{p}}}\left(\hat{Y}_{t}-\hat{A}_{t}\right) & =\mu_{t}
\end{aligned}
$$

Finally we obtain a targeting rule for the pseudo optimal policy.

$$
-\psi_{p} \hat{\Pi}_{t}-\frac{1}{\frac{\theta_{p}}{\psi_{p}}}\left(\hat{Y}_{t}-\hat{A}_{t}\right)+\frac{1}{\frac{\theta_{p}}{\psi_{p}}}\left(\hat{Y}_{t-1}-\hat{A}_{t-1}\right)=0
$$

\section{A.4 Wage Rigidity}

In this Section, we extend our analysis in an economy with sticky wages. Specifically, we assume that nominal wages are a geometric combination of past nominal wages and the wage rate that would prevail in the absence of rigidities.

$$
\begin{aligned}
W_{t} & =W_{t-1}^{\psi_{w}} W_{t}^{* 1-\psi_{w}} \\
W_{t}^{*} & =\chi N_{t}^{\phi} Y_{t} P_{t}
\end{aligned}
$$

The wage conditions can be written in real terms.

$$
\begin{aligned}
w_{t} & =w_{t-1}^{\psi_{w}} \Pi_{t}^{-\psi_{w}} w_{t}^{* 1-\psi_{w}} \\
w_{t}^{*} & =\chi N_{t}^{\phi} Y_{t}
\end{aligned}
$$

Notice that this disconnects the relationship between labor share and output gap. The linearization of the two labor wages equations follow.

$$
\begin{aligned}
\hat{w}_{t} & =\psi_{w} \hat{w}_{t-1}-\psi_{w} \hat{\Pi}_{t}+\left(1-\psi_{w}\right) \hat{w}_{t}^{*} \\
\hat{w}_{t}^{*} & =\phi \hat{N}_{t}+\hat{Y}_{t}
\end{aligned}
$$

\section{A.4.1 Welfare Approximation}

We start from Equation (33).

$$
U_{t}=-\frac{1}{2} \psi_{p} \hat{\Pi}_{t}^{2}-\frac{1}{2} \tilde{\lambda}(1-\tilde{\lambda}) \hat{\Delta}_{c t}^{2}-\frac{1}{2} \frac{1+\phi}{1-\alpha}\left(\hat{Y}_{t}-\hat{A}_{t}\right)^{2}+\frac{(\lambda-\tilde{\lambda})}{1-\tilde{\lambda}}\left[\hat{\tilde{C}}_{k t}+\frac{1}{2} \frac{\tilde{\lambda}}{1-\tilde{\lambda}} \hat{\tilde{C}}_{k t}^{2}+\frac{1}{2} \psi_{p} \hat{\Pi}_{t}^{2}\right]
$$


We also report the equations for $\hat{\tilde{C}}_{k t}$.

$$
\begin{aligned}
\hat{\tilde{C}}_{k t} & =\frac{(1-\alpha)[1-(1-\tau) \delta]}{\tilde{C}_{k}} \hat{L} S_{t}+\left\{\frac{1}{2} \frac{[1-(1-\tau) \delta](1-\alpha)}{\tilde{C}_{k}}\left[1-\frac{[1-(1-\tau) \delta](1-\alpha)}{\tilde{C}_{k}}\right]\right\} \hat{L} \hat{S}_{t}^{2}+ \\
& -\frac{1}{2} \frac{(1-\tau) \delta \psi_{p}}{\tilde{C}_{k}} \hat{\Pi}_{t}^{2}-\gamma \frac{1-\alpha}{\tilde{C}_{k}}\left[1-\frac{[1-(1-\tau) \delta](1-\alpha)}{\tilde{C}_{k}}\right] \hat{A_{t}} \hat{L S}_{t} \\
\hat{\tilde{C}}_{k t}^{2} & =\left[\frac{(1-\alpha)[1-(1-\tau) \delta]}{\tilde{C}_{k}}\right]^{2} \hat{L}_{t}^{2}-2 \gamma \frac{1-\alpha}{\tilde{C}_{k}} \frac{[1-(1-\tau) \delta](1-\alpha)}{\tilde{C}_{k}} \hat{A}_{t} \hat{L S}{ }_{t}
\end{aligned}
$$

The substitution of those expressions into the welfare function yields the following.

$$
\begin{aligned}
U_{t} & =-\frac{1}{2} \psi_{p} \hat{\Pi}_{t}^{2}-\frac{1}{2} \tilde{\lambda}(1-\tilde{\lambda}) \hat{\Delta}_{c t}^{2}-\frac{1}{2} \frac{1+\phi}{1-\alpha}\left(\hat{Y}_{t}-\hat{A}_{t}\right)^{2}+\frac{(\lambda-\tilde{\lambda})}{1-\tilde{\lambda}}\left[\frac{(1-\alpha)[1-(1-\tau) \delta]}{\tilde{C}_{k}} \hat{L}_{t}\right]+ \\
& +\frac{(\lambda-\tilde{\lambda})}{1-\tilde{\lambda}}\left[\frac{1}{2} \frac{[1-(1-\tau) \delta](1-\alpha)}{\tilde{C}_{k}}\left[1-\frac{[1-(1-\tau) \delta](1-\alpha)}{\tilde{C}_{k}}\right] \hat{\left.L S_{t}^{2}\right]+}\right. \\
& +\frac{(\lambda-\tilde{\lambda})}{1-\tilde{\lambda}}\left[-\frac{1}{2} \frac{(1-\tau) \delta \psi_{p}}{\tilde{C}_{k}} \hat{\Pi}_{t}^{2}-\gamma \frac{1-\alpha}{\tilde{C}_{k}}\left[1-\frac{[1-(1-\tau) \delta](1-\alpha)}{\tilde{C}_{k}}\right] \hat{\hat{A}_{t}} \hat{\left.L S_{t}\right]+}\right. \\
& +\frac{(\lambda-\tilde{\lambda})}{1-\tilde{\lambda}} \frac{1}{2} \frac{\tilde{\lambda}}{1-\tilde{\lambda}}\left[\left[\frac{(1-\alpha)[1-(1-\tau) \delta]}{\tilde{\tilde{C}}_{k}}\right]^{2} \hat{L}_{t}^{2}-2 \gamma \frac{1-\alpha}{\tilde{C}_{k}} \frac{[1-(1-\tau) \delta](1-\alpha)}{\tilde{\tilde{C}}_{k}} \hat{A}_{t} \hat{L S_{t}}\right] \\
& +\frac{(\lambda-\tilde{\lambda})}{1-\tilde{\lambda}}\left[\frac{1}{2} \psi_{p} \hat{\Pi}_{t}^{2}\right]
\end{aligned}
$$


We can collect terms and have the following coefficients (for completeness, we report all the coefficients again).

$$
\begin{aligned}
& \hat{\Pi}_{t}^{2}:-\frac{1}{2} \psi_{p}\left[1-\frac{(\lambda-\tilde{\lambda})}{1-\tilde{\lambda}}\left[1-\frac{(1-\tau) \delta}{\tilde{C}_{k}}\right]\right] \\
& \hat{\Delta}_{c t}^{2}:-\frac{1}{2} \tilde{\lambda}(1-\tilde{\lambda}) \\
& \hat{Y}_{t}^{2}:-\frac{1}{2} \frac{1+\phi}{1-\alpha} \\
& \hat{L S_{t}^{2}}: \frac{1}{2} \frac{(\lambda-\tilde{\lambda})}{1-\tilde{\lambda}} \frac{[1-(1-\tau) \delta](1-\alpha)}{\tilde{\tilde{C}}_{k}}\left[1-\frac{1-2 \tilde{\lambda}}{1-\tilde{\lambda}} \frac{[1-(1-\tau) \delta](1-\alpha)}{\tilde{\tilde{C}}_{k}}\right] \\
& \hat{A}_{t} \hat{Y}_{t}: \frac{1+\phi}{1-\alpha} \\
& \hat{A}_{t} \hat{L S_{t}}:-\frac{(\lambda-\tilde{\lambda})}{1-\tilde{\lambda}} \gamma \frac{1-\alpha}{\tilde{\tilde{C}}_{k}}\left[1-\frac{1-2 \tilde{\lambda}}{1-\tilde{\lambda}} \frac{[1-(1-\tau) \delta](1-\alpha)}{\tilde{\tilde{C}}_{k}}\right] \\
& \hat{L S_{t}}: \frac{(\lambda-\tilde{\lambda})}{1-\tilde{\lambda}} \frac{[1-(1-\tau) \delta](1-\alpha)}{\tilde{C}_{k}} \\
& \mathbf{W}=\sum_{t=0}^{\infty} \beta^{t}\left\{-\frac{1}{2} W_{\Pi} \hat{\Pi}_{t}^{2}-\frac{1}{2} W_{\Delta_{c}} \hat{\Delta}_{t}^{2}-\frac{1}{2} \frac{1+\phi}{1-\alpha}\left(\hat{Y}_{t}-\hat{A}_{t}\right)^{2}+\frac{1}{2} \tilde{W}_{L S^{2}} \hat{L S_{t}^{2}}+W_{A L S} \hat{A}_{t} \hat{L S_{t}}+W_{L S} \hat{L S_{t}}\right\} \\
& \equiv-\frac{1}{2} W_{\Pi} \\
& \equiv-\frac{1}{2} W_{\Delta_{c}} \\
& \equiv-\frac{1}{2} \tilde{W}_{Y^{2}} \\
& \equiv \frac{1}{2} \tilde{W}_{L S^{2}} \\
& \equiv W_{A Y} \\
& \equiv W_{A L S} \\
& \equiv W_{L S}
\end{aligned}
$$

We report the New Keynesian Phillips curve second order approximation.

$$
\sum_{t=0}^{\infty} \beta^{t} \hat{L S} t=\frac{\psi_{p}}{\theta_{p}} T_{0}+\sum_{t=0}^{\infty} \beta^{t}\left\{-\left(\frac{1}{2}+\frac{\tilde{\lambda}}{1-\tilde{\lambda}} \frac{(1-\alpha)(1-(1-\tau) \delta)}{\tilde{\tilde{C}}_{k}}\right) \hat{L S_{t}^{2}}+\frac{\tilde{\lambda}}{1-\tilde{\lambda}} \gamma \frac{(1-\alpha)}{\tilde{\tilde{C}}_{k}} \hat{A}_{t} \hat{L S_{t}}\right\}
$$

Multiplying the above expression by $\frac{(\lambda-\tilde{\lambda})}{1-\tilde{\lambda}} \frac{[1-(1-\tau) \delta](1-\alpha)}{\tilde{C}_{k}}$. This transforms the Phillips curve in a very convenient form.

$$
\begin{aligned}
\sum_{t=0}^{\infty} \beta^{t} W_{L S} \hat{L} S_{t} & =\frac{\psi_{p}}{\theta_{p}} \frac{(\lambda-\tilde{\lambda})}{1-\tilde{\lambda}} \frac{[1-(1-\tau) \delta](1-\alpha)}{\tilde{C}_{k}} \tilde{T}_{0}+ \\
& +\frac{(\lambda-\tilde{\lambda})}{1-\tilde{\lambda}} \frac{[1-(1-\tau) \delta](1-\alpha)}{\tilde{\tilde{C}}_{k}} \sum_{t=0}^{\infty} \beta^{t}\left\{-\left(\frac{1}{2}+\frac{\tilde{\lambda}}{1-\tilde{\lambda}} \frac{(1-\alpha)(1-(1-\tau) \delta)}{\tilde{C}_{k}}\right) \hat{L S_{t}^{2}}\right\} \\
& +\frac{(\lambda-\tilde{\lambda})}{1-\tilde{\lambda}} \frac{[1-(1-\tau) \delta](1-\alpha)}{\tilde{\tilde{C}}_{k}} \sum_{t=0}^{\infty} \beta^{t}\left\{\frac{\tilde{\lambda}}{1-\tilde{\lambda}} \gamma \frac{(1-\alpha)}{\tilde{\tilde{C}}_{k}} \hat{A}_{t} \hat{L S_{t}}\right\}
\end{aligned}
$$


Finally, the welfare function becomes the following.

$$
\begin{aligned}
\mathbf{W} & =\sum_{t=0}^{\infty} \beta^{t}\left\{-\frac{1}{2} W_{\Pi} \hat{\Pi}_{t}^{2}-\frac{1}{2} W_{\Delta_{c}} \hat{\Delta}_{t}^{2}-\frac{1}{2} \frac{1+\phi}{1-\alpha}\left(\hat{Y}_{t}-\hat{A}_{t}\right)^{2}+\frac{1}{2} \tilde{W}_{L S^{2}} \hat{L S}_{t}^{2}+W_{A L S} \hat{A}_{t} \hat{L S} \hat{S}_{t}\right\}+T_{0}+ \\
& +\frac{(\lambda-\tilde{\lambda})}{1-\tilde{\lambda}} \frac{[1-(1-\tau) \delta](1-\alpha)}{\tilde{\tilde{C}}_{k}} \sum_{t=0}^{\infty} \beta^{t}\left\{-\left(\frac{1}{2}+\frac{\tilde{\lambda}}{1-\tilde{\lambda}} \frac{(1-\alpha)(1-(1-\tau) \delta)}{\tilde{C}_{k}}\right) \hat{L S_{t}^{2}}\right\} \\
& +\frac{(\lambda-\tilde{\lambda})}{1-\tilde{\lambda}} \frac{[1-(1-\tau) \delta](1-\alpha)}{\tilde{C}_{k}} \sum_{t=0}^{\infty} \beta^{t}\left\{\frac{\tilde{\lambda}}{1-\tilde{\lambda}} \gamma \frac{(1-\alpha)}{\tilde{C}_{k}} \hat{A}_{t} \hat{L S}\right\}
\end{aligned}
$$

Collecting terms we have the following.

$$
\mathbf{W}=\sum_{t=0}^{\infty} \beta^{t}\left\{-\frac{1}{2} W_{\Pi} \hat{\Pi}_{t}^{2}-\frac{1}{2} W_{\Delta_{c}} \hat{\Delta}_{t}^{2}-\frac{1}{2} \frac{1+\phi}{1-\alpha}\left(\hat{Y}_{t}-\hat{A}_{t}\right)^{2}-\frac{1}{2} W_{L S^{2}}\left(\hat{L} S_{t}-\hat{L} \hat{S}_{t}^{*}\right)^{2}\right\}+T_{0}
$$

where

$$
\begin{aligned}
W_{L S^{2}} & =\frac{\lambda-\tilde{\lambda}}{(1-\tilde{\lambda})^{2}}\left(\frac{1-(1-\tau) \delta}{\tilde{C}_{k}}(1-\alpha)\right)^{2} \\
\hat{L} S_{t}^{*} & =-\frac{(1-\tilde{\lambda}) \gamma\left(1-\frac{[1-(1-\tau) \delta](1-\alpha)}{\tilde{\tilde{C}}_{k}}\right)}{\frac{[1-(1-\tau) \delta]^{2}(1-\alpha)}{\tilde{C}_{k}}} \hat{A}_{t}
\end{aligned}
$$

\section{A.4.2 Optimal Monetary Policy Under Wage Rigidities}

The Lagrangian of the maximization problem is the following:

$$
\begin{aligned}
\mathbf{L} & =\sum_{t=0}^{\infty} \beta^{t}\left\{-\frac{1}{2} W_{\Pi} \hat{\Pi}_{t}^{2}-\frac{1}{2} W_{\Delta_{c}} \hat{\Delta}_{t}^{2}-\frac{1}{2} \frac{1+\phi}{1-\alpha}\left(\hat{Y}_{t}-\hat{A}_{t}\right)^{2}-\frac{1}{2} W_{L S^{2}}\left(\hat{L S} S_{t}-\hat{L} S_{t}^{*}\right)^{2}\right\}+T_{0}+ \\
& +\sum_{t=0}^{\infty} \beta^{t} \mu_{t}\left[\hat{\Pi}_{t}-\beta E_{t} \hat{\Pi}_{t+1}-\frac{\theta_{p}}{\psi_{p}}\left(\hat{L S} \hat{S}_{t}-\hat{L S} S_{t}^{*}\right]+\right. \\
& +\sum_{t=0}^{\infty} \beta^{t} \eta_{t}\left[\hat{\Delta}_{t}+\frac{1}{1-\tilde{\lambda}} \frac{1-(1-\tau) \delta}{\tilde{C}_{k}}(1-\alpha) \frac{1+\phi}{1-\alpha}\left(\hat{Y}_{t}-\hat{A}_{t}\right)\right]+ \\
& +\sum_{t=0}^{\infty} \beta^{t} \psi_{t}\left[\hat{L} S_{t}-\hat{w}_{t}-\frac{\alpha}{1-\alpha} \hat{Y}_{t}-\frac{1}{1-\alpha} \hat{A}_{t}\right]+ \\
& +\sum_{t=0}^{\infty} \beta^{t} \nu_{t}\left[\hat{w}_{t}-\psi_{w} \hat{w}_{t-1}+\psi_{w} \hat{\Pi}_{t}-\left(1-\psi_{w}\right) \frac{1-\alpha+\phi}{1-\alpha} \hat{Y}_{t}+\left(1-\psi_{w}\right) \frac{\phi}{1-\alpha} \hat{A}_{t}\right]
\end{aligned}
$$


The first order conditions follow.

$$
\begin{array}{r}
-\beta^{t} W_{\Pi} \hat{\Pi}_{t}+\beta^{t} \mu_{t}-\beta^{t} \mu_{t-1}+\beta^{t} \nu_{t} \psi_{w}=0 \\
-\beta^{t} W_{\Delta_{c}} \hat{\Delta}_{t}+\beta^{t} \eta_{t}=0 \\
-\beta^{t} \frac{1+\phi}{1-\alpha}\left(\hat{Y}_{t}-\hat{A}_{t}\right)+\beta^{t} \eta_{t} \frac{1}{1-\tilde{\lambda}} \frac{1-(1-\tau) \delta}{\tilde{C}_{k}}(1-\alpha) \frac{1+\phi}{1-\alpha}-\beta^{t} \psi_{t} \frac{\alpha}{1-\alpha}-\beta^{t} \nu_{t}\left(1-\psi_{w}\right) \frac{1-\alpha+\phi}{1-\alpha}=0 \\
-\beta^{t} W_{L S^{2}}\left(\hat{L} S_{t}-\hat{L} S_{t}^{*}\right)-\beta^{t} \mu_{t} \frac{\theta_{p}}{\psi_{p}}+\beta^{t} \psi_{t}=0 \\
-\beta^{t} \psi_{t}+\beta^{t} \nu_{t}-\beta^{t+1} \nu_{t+1} \psi_{w}=0
\end{array}
$$

The system of equations can be written as

$$
\begin{aligned}
&-W_{\Pi} \hat{\Pi}_{t}+\mu_{t}-\mu_{t-1}+\nu_{t} \psi_{w}=0 \\
&-W_{\Delta_{c}} \hat{\Delta}_{t}+\eta_{t}=0 \\
&-\frac{1+\phi}{1-\alpha}\left(\hat{Y}_{t}-\hat{A}_{t}\right)+\eta_{t} \frac{1}{1-\tilde{\lambda}} \frac{1-(1-\tau) \delta}{\tilde{C}_{k}}(1-\alpha) \frac{1+\phi}{1-\alpha}-\psi_{t} \frac{\alpha}{1-\alpha}-\nu_{t}\left(1-\psi_{w}\right) \frac{1-\alpha+\phi}{1-\alpha}=0 \\
&-W_{L S^{2}}\left(\hat{L} S_{t}-\hat{L} S_{t}^{*}\right)-\mu_{t} \frac{\theta_{p}}{\psi_{p}}+\psi_{t}=0 \\
&-\psi_{t}+\nu_{t}-\beta \nu_{t+1} \psi_{w}=0
\end{aligned}
$$

\section{A.4.3 RANK-Optimal Monetary Policy Under Wage Rigidities}

The RANK-optimal monetary policy problem is one where again the central bank believes $\lambda=0$, implying that $W_{\Pi}=\psi_{p}$ as well as $W_{L S^{2}}=0$ in the above equations for optimal monetary policy.

\section{A.4.4 Results of Model under Wage Rigidities}

In the model with wage rigidities, the calibration follows the steps as if wage were flexible. We pick $\psi_{w}=$ $0.75,{ }^{33}$ meaning that only one quarter of the gap between flex and rigid wage is closed every period. We calibrate then $\gamma$ in the same way as described in Section 2.12, and obtain 2.27.

Below we report the results of all the exercises under the wage rigidity exercise.

\footnotetext{
${ }^{33}$ This value is taken from the literature, see Galí, 2015. In "Calvo" setting, this would correspond to readjusting nominal wages with a 0.25 probability at each quarter.
} 
Figure A1: Welfare Weights and Redistribution Parameter $\tau$

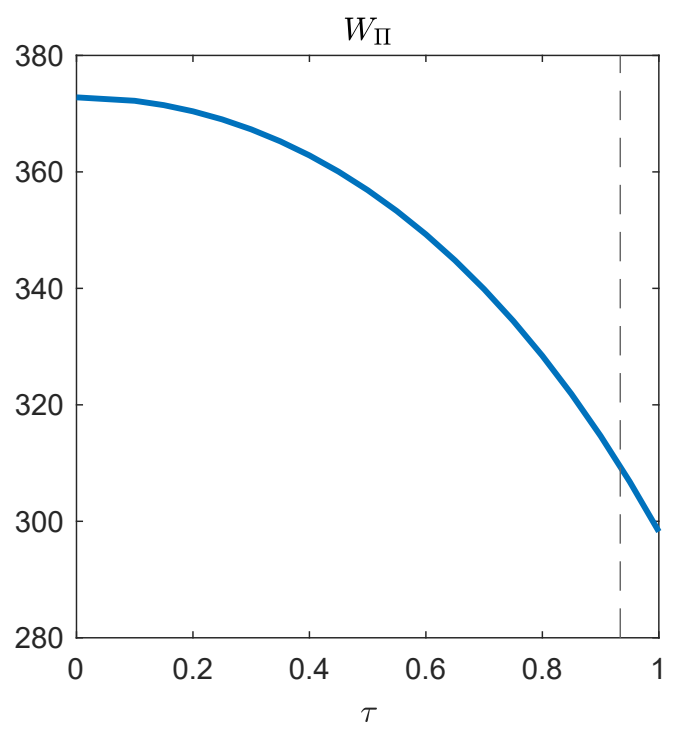

(a) Weight on Inflation Gap

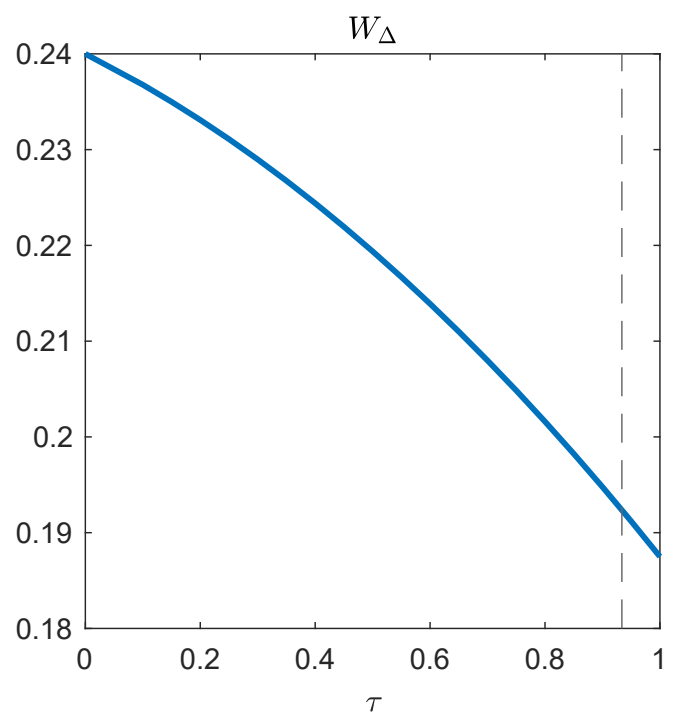

(c) Weight on Consumption Inequality Gap

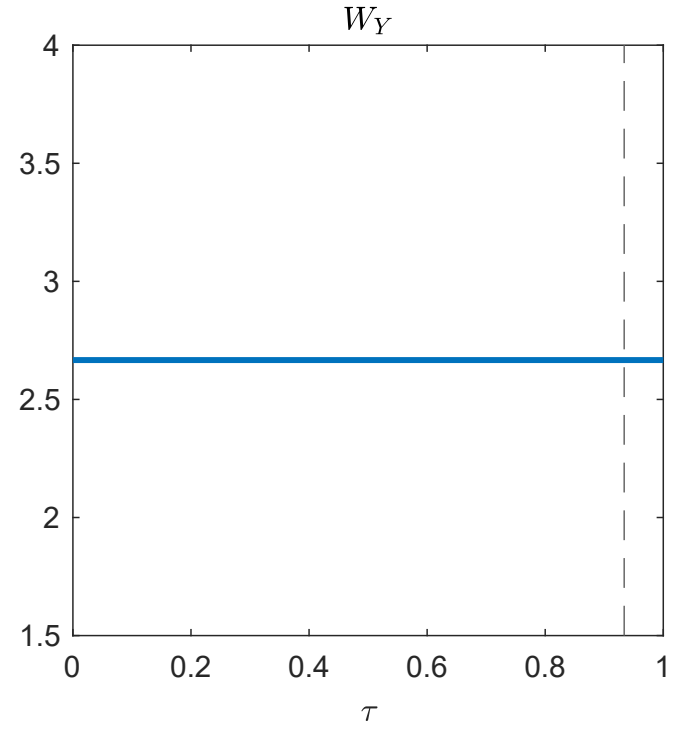

(b) Weight on Output Gap

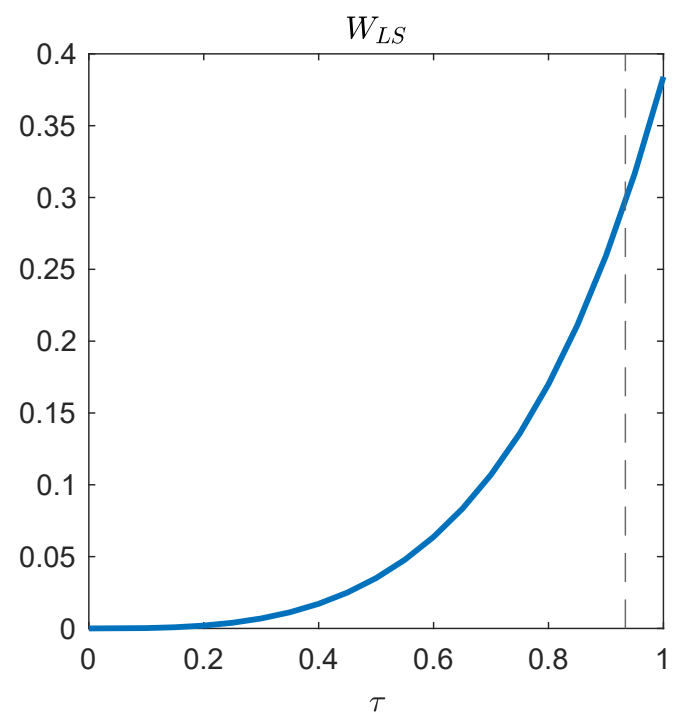

(d) Weight on Labor Share Gap

Notes: The panels show the four weights as a function of the extent to which dividends are redistributed, governed by $\tau$. All other parameters are set according to the baseline calibration in Table 1. 
Figure A2: Impulse Response to a Positive TFP shock: Optimal vs RANK-Optimal Policy, with Wage Rigidity

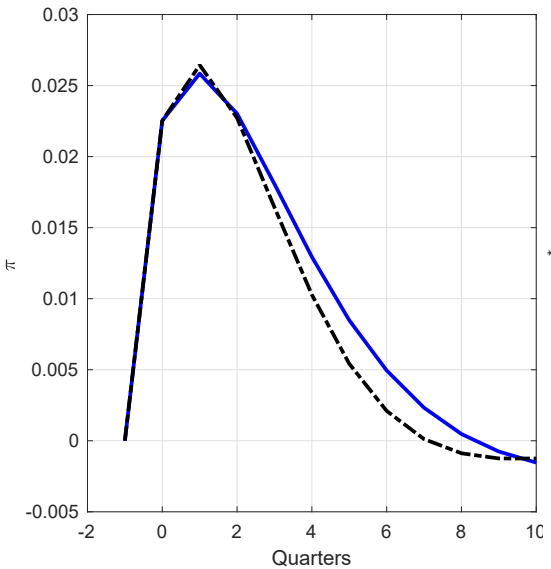

(a.) Inflation

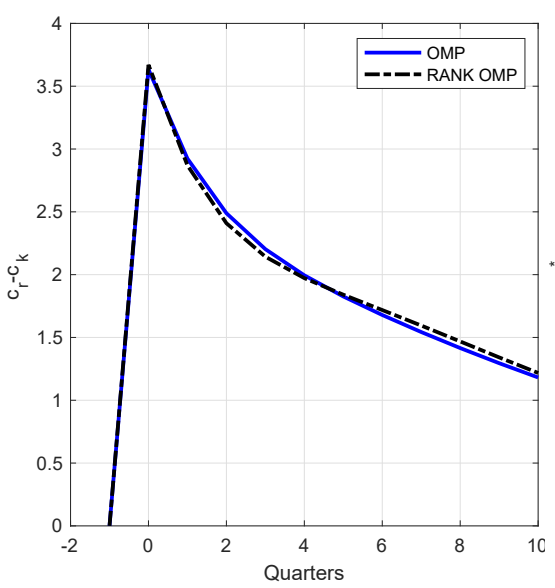

(c) Inequality

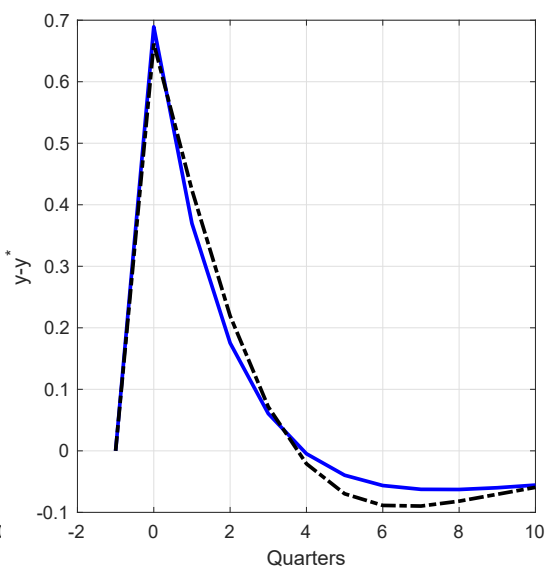

(b) Output Gap

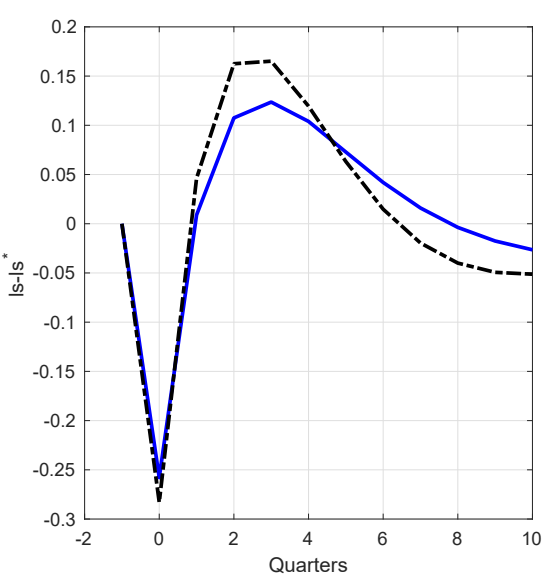

(d) Labor Share

Notes: The four panels show the impulse response functions of inflation, output gap, consumption inequality, and labor share respectively, in percentage points under the fully optimal policy (blue) and the RANK-optimal policy (black dash-dotted).

The y-axis measure the deviation from each variable's steady state, measured in percentage points.

Table A1: Welfare in Taylor Rules - Standard Parametrization

\begin{tabular}{c|c|c|c|c|c|c|c|c} 
& $(1)$ & $(2)$ & $(3)$ & $(4)$ & $(5)$ & $(6)$ & $(7)$ & $(8)$ \\
\hline \hline$\tau$ & 0 & 0 & 0 & 0 & 0.9 & 0.9 & 0.9 & 0.9 \\
$\gamma$ & 0 & 0 & 0 & 0 & 2.2 & 2.2 & 2.2 & 2.2 \\
$\phi_{\pi}$ & 1.5 & 1.5 & 5 & 1.5 & 1.5 & 1.5 & 5 & 1.5 \\
$\phi_{y}$ & 0.1 & 0 & 0 & 1 & 0.1 & 0 & 0 & 1 \\
\hline Cons. Equiv. Loss & 0.2 & 0.1 & 0 & 0.7 & 2.3 & 2 & 0.6 & 2.8 \\
Cons. Equiv. $W-T_{0}$ & 0.3 & 0.1 & 0 & 0.9 & 0.8 & 0.6 & 0.1 & 1.1 \\
Inflation & 94.8 & 94.7 & 91.1 & 94.8 & 78.1 & 75.8 & 23.4 & 81 \\
Output & 5.2 & 5.3 & 8.9 & 5.2 & 5 & 4.9 & 1.6 & 5.2 \\
Inequality & 0 & 0 & 0 & 0 & 16.1 & 18.8 & 72.9 & 12.7 \\
Labor Share & 0 & 0 & 0 & 0 & 0.8 & 0.6 & 2.1 & 1.1 \\
\hline \hline
\end{tabular}

Notes: The table reports 1) the consumption equivalent value $x$, multiplied by 10000 (e.g. 1 means 0.0001 percent of steady state consumption); 2) the dynamically relevant welfare component; 3) the inflation share of welfare loss; 4) the output share of welfare loss; 5) the inequality share of welfare loss; and 6) the labor share part of welfare loss. 
Figure A3: Welfare of Optimal vs. RANK-Optimal Policy in Consumption Equivalent Terms
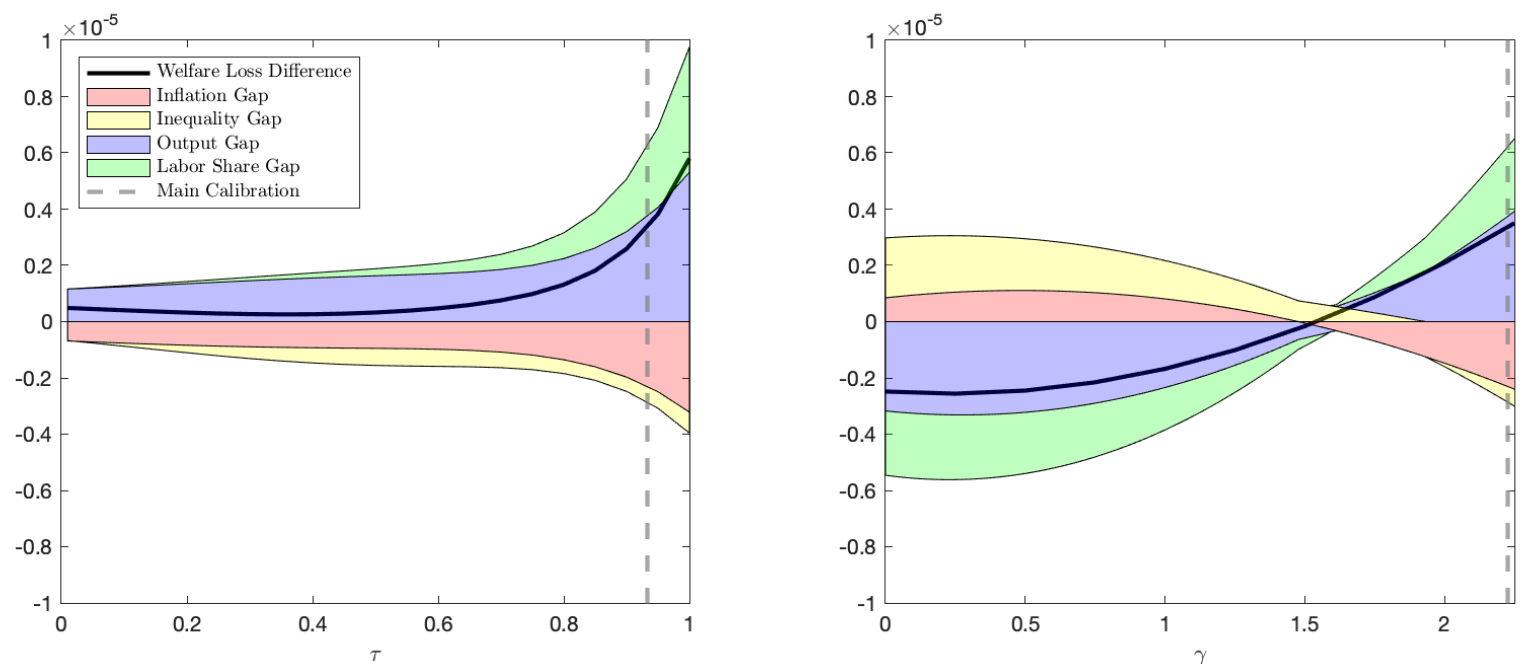

Notes: The panels show the difference of the welfare functions between Optimal Policy and Rank Optimal Policy as functions of parameters. Shaded areas represent the differences by gaps (inflation, output gap, and inequality). The x-axis on left (right) panel represents $\tau(\gamma)$. The vertical dashed line denotes our baseline calibration.

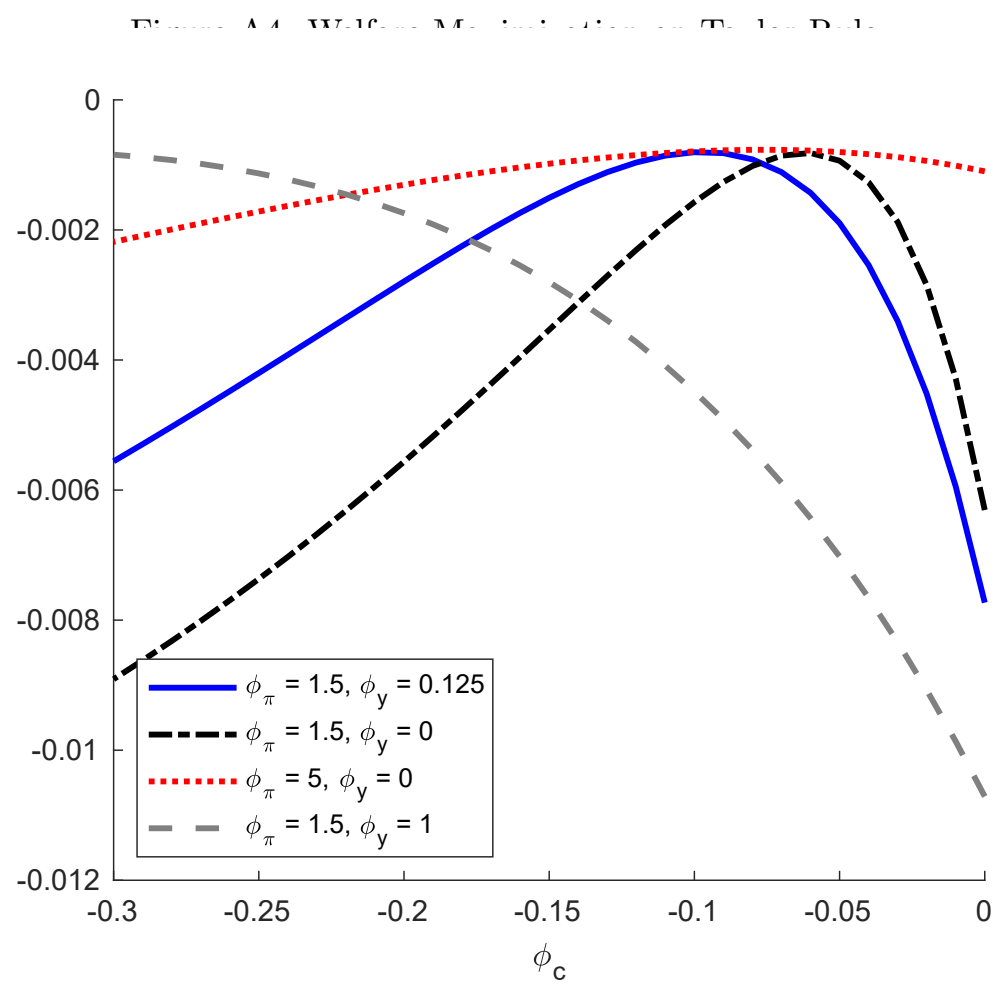

Notes: The figure shows how welfare changes depending on the Taylor rule parameter on consumption inequality, $\phi_{c}$. Each line corresponds to the four rules evaluated in Table 2, that use a different combination of Taylor rule parameters on inflation, $\phi_{\pi}$, and output, $\phi_{y}$. These are all under our baseline calibration, including using $\tau=0.93$ and $\gamma=1.67$. 
Figure A5: Impulse Response Functions: Standard and Augmented Taylor rules - Wage Rigidity

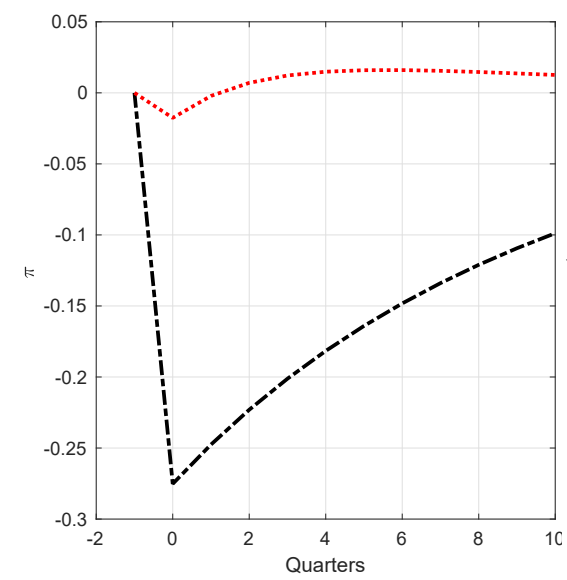

(a.) Inflation

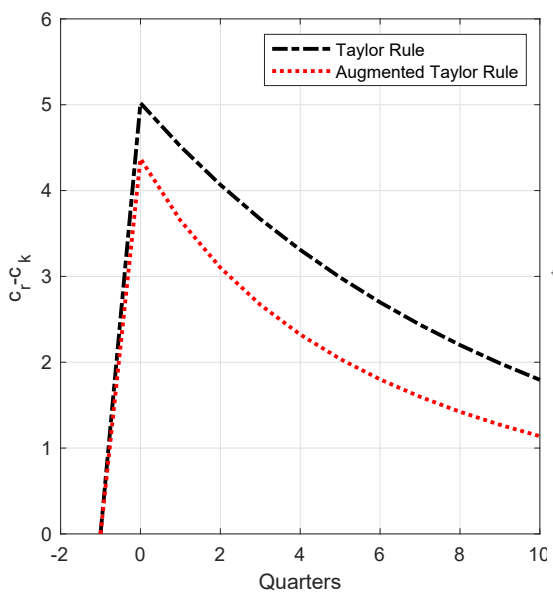

(c) Inequality

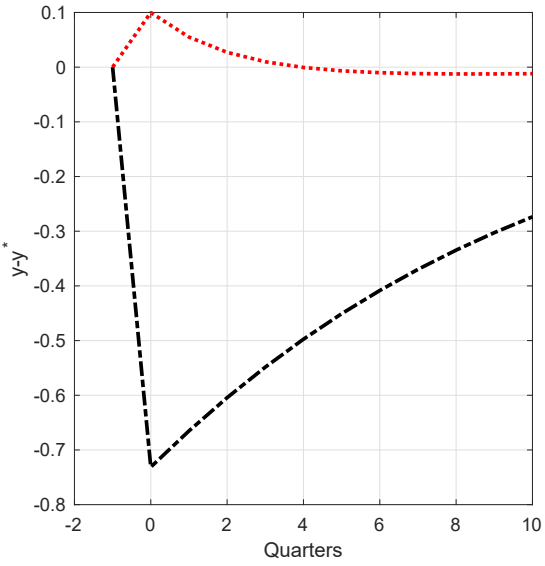

(b) Output Gap

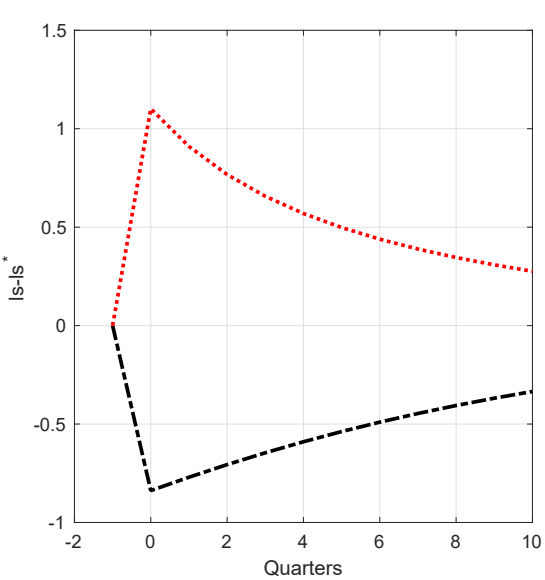

(d) Labor Share

Notes: The four panels show the Impulse Response Function of, respectively, inflation, welfare relevant output gap, consumption inequality gap, and labor share. The two lines correspond to two different policies under our baseline calibration. The black dashed-dotted line corresponds to the Standard Taylor rule using $\phi_{\pi}=1.5, \phi_{y}=0.125$ and $\phi_{c}=0$, and the red dotted line corresponds to the Augmented Taylor Rule where $\phi_{\pi}=1.5, \phi_{y}=0.125, \phi_{c}=\phi_{c}^{*}$. 